ARTICLE

\title{
GDF11 decreases bone mass by stimulating osteoclastogenesis and inhibiting osteoblast differentiation
}

Weiqing Liu ${ }^{1, \star}$, Liyan Zhou ${ }^{1, \star}$, Chenchen Zhou ${ }^{1, \star}$, Shiwen Zhang ${ }^{1}$, Junjun Jing ${ }^{1}$, Liang Xie ${ }^{1}$, Ningyuan Sun ${ }^{1}$, Xiaobo Duan ${ }^{1}$, Wei Jing ${ }^{1}$, Xing Liang ${ }^{1}$, Hu Zhao ${ }^{1}$, Ling Ye ${ }^{1}$, Qianming Chen ${ }^{1} \&$ Quan Yuan ${ }^{1}$

Osteoporosis is an age-related disease that affects millions of people. Growth differentiation factor 11 (GDF11) is a secreted member of the transforming growth factor beta (TGF- $\beta$ ) superfamily. Deletion of Gdf11 has been shown to result in a skeletal anterior-posterior patterning disorder. Here we show a role for GDF11 in bone remodelling. GDF11 treatment leads to bone loss in both young and aged mice. GDF11 inhibits osteoblast differentiation and also stimulates RANKL-induced osteoclastogenesis through Smad2/3 and c-Fos-dependent induction of Nfatc1. Injection of GDF11 impairs bone regeneration in mice and blocking GDF11 function prevents oestrogen-deficiency-induced bone loss and ameliorates age-related osteoporosis. Our data demonstrate that GDF11 is a previously unrecognized regulator of bone remodelling and suggest that GDF11 is a potential target for treatment of osteoporosis.

\footnotetext{
${ }^{1}$ State Key Laboratory of Oral diseases, West China Hospital of Stomatology, Sichuan University, Chengdu 610041, China. ${ }^{*}$ These authors contributed equally to this work. Correspondence and requests for materials should be addressed to Q.Y. (email: yuanquan@scu.edu.cn).
} 
O steoporosis is a skeletal disorder that affects millions of individuals, especially older and postmenopausal women ${ }^{1,2}$. The skeleton is continuously remodelled by a process in which pre-existing bone is removed by osteoclasts, specialized multinucleated cells derived from haematopoietic precursors, and rebuilt by osteoblasts derived from mesenchymal stem cells ${ }^{3-5}$. Bone loss occurs when the proper balance or bone formation and bone resorption is disrupted. Local cytokines and growth factors secreted by bone cells, as well as systemic hormones, have important roles in maintaining this critical balance ${ }^{6-8}$.

GDF11 is a secreted member of the transforming growth factor beta (TGF- $\beta$ ) superfamily ${ }^{9}$, several members of which have been implicated in regulating bone remodelling ${ }^{10}$. For example, TGF- $\beta$ induces osteoclast formation ${ }^{11,12}$ and represses osteoblast differentiation ${ }^{13-15}$. Myostatin, which is highly homologous with GDF11 (ref. 9), was shown to induce osteoclastogenesis ${ }^{16}$. The direct role of GDF11 in bone development has been demonstrated in knockout mice that display skeletal patterning problems related to anterior-posterior positioning ${ }^{17,18}$. Repression of GDF11 function by transgenic overexpression of skeletal GDF11 propeptide results in an increase in bone mass ${ }^{19}$. GDF11 also regulates the differentiation of odontoblasts and repair of injured dentin ${ }^{20,21}$. However, the possible function of GDF11 in postnatal bone remodelling in vivo is unclear.

Some studies suggest that GDF11 acts as an anti-ageing factor $^{22-25}$. Serum levels of GDF11 decline with age and injection of recombinant GDF11 (rGDF11) seems to reverse age-related dysfunction in brain, heart and skeletal muscle by rejuvenating stem cells ${ }^{22-25}$. However, other studies demonstrate opposite results, showing that GDF11 level does not change or increase with age ${ }^{26,27}$, and elevated GDF11 is a risk factor for age-related frailty and disease in humans ${ }^{26,28}$. Systemic administration of rGDF11 in mice inhibits skeletal muscle regeneration ${ }^{27}$ and fails to rescue age-related pathological cardiac hypertrophy ${ }^{29}$. These conflicting results led us to study whether GDF11 is associated with age-related bone loss. Here we show that injection of rGDF11 results in bone loss, as well as inhibition of bone regeneration in both young and aged mice. Importantly, the delivery of a GDF11 blocking antibody prevents oestrogendeficiency-induced bone loss and ameliorates age-related osteoporosis, implying that GDF11 inhibition might be a potential therapeutic approach to prevent osteoporotic bone loss.

\section{Results}

GDF11 treatment leads to bone loss in young adult mice. We first examined the role of GDF11 in bone remodelling by administering young adult mice ( 9 week old) with daily intraperitoneal injections of rGDF11 ( 0.1 or $0.3 \mathrm{mg} \mathrm{kg}^{-1}$ body weight; $\# 120$-11, PeproTech, Rocky Hill, NJ) or vehicle (saline) for 6 weeks. MicroCT analysis of the secondary spongiosa of the distal femur metaphysis revealed that the trabecular bone volume $(\mathrm{BV} / \mathrm{TV})$ in mice treated with the higher dose of rGDF11 $\left(0.3 \mathrm{mg} \mathrm{kg}^{-1}\right)$ was significantly lower than in mice treated with vehicle (Fig. 1a,d). Mice given the lower dose of rGDF11 $\left(0.1 \mathrm{mg} \mathrm{kg}^{-1}\right)$ also showed a trend of decreased trabecular bone volume, although it was not statistically significant $(P=0.078)$. Von Kossa staining further confirmed the loss of trabecular bone in high-dose rGDF11 mice (Fig. 1b). Histomorphometric analysis revealed a significant increase in osteoclast number (N.Oc/B.Pm) and a decrease in osteoblast number (N.Ob/B.Pm; Fig. 1c,d). Meanwhile, the mineral apposition rate (MAR) and bone formation rate $(\mathrm{BFR} / \mathrm{BS})$ in the high-dose $\mathrm{rGDF} 11$ mice were both significantly lower than the rates in vehicle-treated controls (Fig. 1d), indicating a reduction in bone formation. We also performed enzyme-linked immunosorbent assay (ELISA) to assess the serum markers for bone turnover. The serum levels of c-terminal telopeptides of collagen type I (CTX), a marker for bone resorption, were significantly higher in high-dose rGDF11 mice (Fig. 1e). In contrast, the serum levels of the bone formation marker P1NP were reduced (Fig. 1f).

GDF11 treatment leads to bone loss in aged mice. Given the controversial role of GDF11 in aging pathologies, we also investigated the effect of rGDF11 in aged mice ( 18 month old) by daily intraperitoneal injections of $0.3 \mathrm{mg} \mathrm{kg}^{-1} \mathrm{rGDF} 11$ for 6 weeks. Consistent with young adult mice, we found that rGDF11 administration led to trabecular bone loss in the distal femur metaphysis of aged animals, with increased osteoclast number (N.Oc/B.Pm) and decreased osteoblast number (N.Ob/B.Pm) (Fig. 2a-d). There is little trabecular bone left in the femur metaphysis of aged mice, so we evaluated the L4 vertebrae and observed similar results (Fig. 2e,f). In addition, the serum levels of CTX were also significantly elevated in rGDF11 treated mice (Fig. 2g).

Previous studies showed that rGDF11 may rejuvenate muscle stem cells ${ }^{24}$. We therefore investigated whether rGDF11 $\left(0.3 \mathrm{mg} \mathrm{kg}^{-1}\right)$ administration might have a similar effect on bone marrow stem cells (BMSCs). However, we observed that the percentage of BMSCs, as determined by flow cytometry, did not change in either young or aged mice after daily treatments for 6 weeks (Supplementary Fig. 1a,b). We compared colony formation in these sorted cells and found no significant differences between cells from treated and control mice (Supplementary Fig. 1c,d). Moreover, the rGDF11 treatment had no apparent effect on the proliferation of these sorted cells (Supplementary Fig. 1e).

GDF11 stimulates RANKL-induced osteoclastogenesis. We next assessed the direct contribution of GDF11 to osteoclastmediated bone resorption. We analysed osteoclast differentiation in vitro and found that rGDF11 alone was not able to induce osteoclastogenesis of bone marrow-derived macrophages (BMMs; Fig. 3a, upper panel). We therefore supplemented medium containing receptor activator of nuclear factor kappa-B ligand (RANKL) with rGDF11. The presence of either $50 \mathrm{ng} \mathrm{ml}^{-1}$ or $100 \mathrm{ng} \mathrm{ml}^{-1}$ rGDF11 in the medium significantly stimulated osteoclast differentiation, as evidenced by an increase of the number of tartrate-resistant acid phosphatase (TRAP) positive multinucleated cells after 4 days of treatment (Fig. 3a lower panel, b). Notably, rGDF11 treatment also resulted in very large osteoclasts with a huge cytoplasmatic compartment, which indicates that GDF11 stimulated osteoclast maturation (Fig. 3c). On the contrary, only a few small osteoclasts were generated in control media during this short test period. The presence of rGDF11 did not significantly affect the proliferation or apoptosis of BMMs (Fig. 3d,e). We next performed resorption pit analyses using the Osseo Assay plate (Corning) and observed a larger number of resorption pits and a larger area overall in the presence of rGDF11 compared with RANKL treatment alone (Fig. 3f-h). Finally, we examined osteoclast formation in a co-culture of BMMs and calvarial osteoblasts to mimic the in vivo environment. The presence of rGDF11 led to an obvious increase of the number and size of TRAP-positive multinucleated cells (Fig. 2i-k).

We further elucidated the molecular mechanism by performing a microarray analysis. Compared with RANKL alone, rGDF11 supplementation increased the expression of 467 genes (Fig. 4a), including $N f a c t c 1$, the master transcriptional factor of osteoclast differentiation, as well as other key marker genes, such as Fos, Src, 


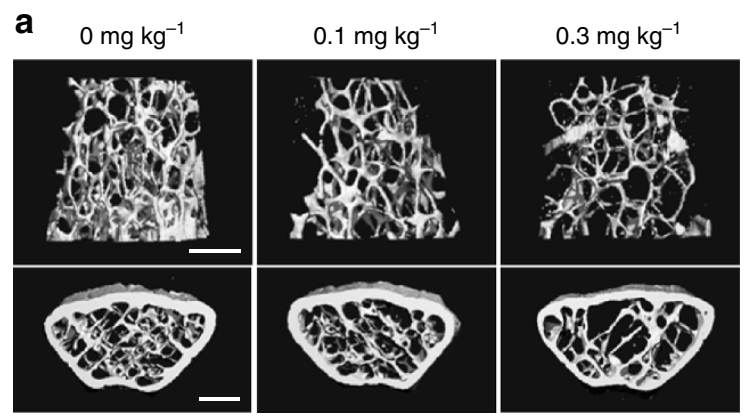

b
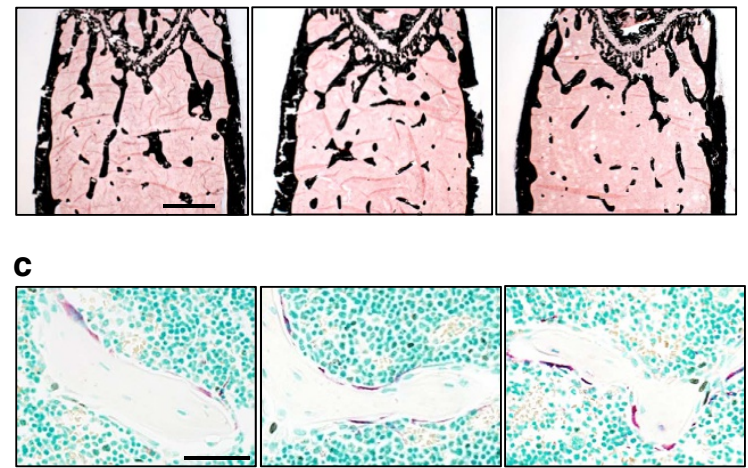

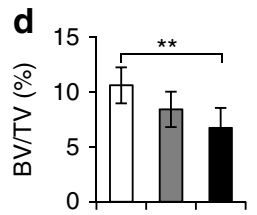

$\begin{array}{llll}\text { rGDF11: } 0 & 0.1 & 0.3\end{array}$ $\left(\mathrm{mg} \mathrm{kg}^{-1}\right)$

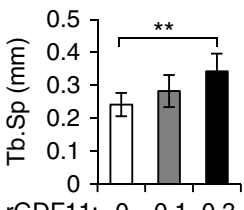

rGDF11: $0 \quad 0.10 .3$ $\left(\mathrm{mg} \mathrm{kg}^{-1}\right)$

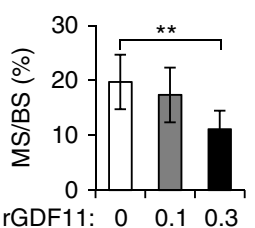
rGDF11:
$\left(\mathrm{mg} \mathrm{kg}^{-1}\right)$

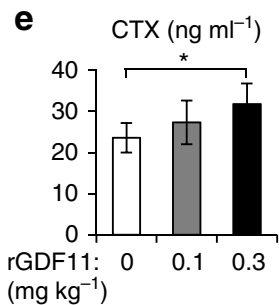

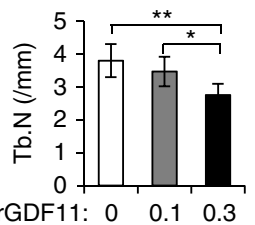

rGDF11:
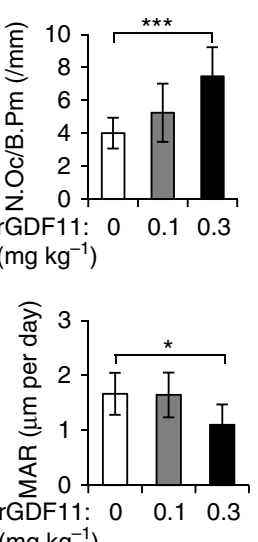

$\left(\mathrm{mg} \mathrm{kg}^{-1}\right)$
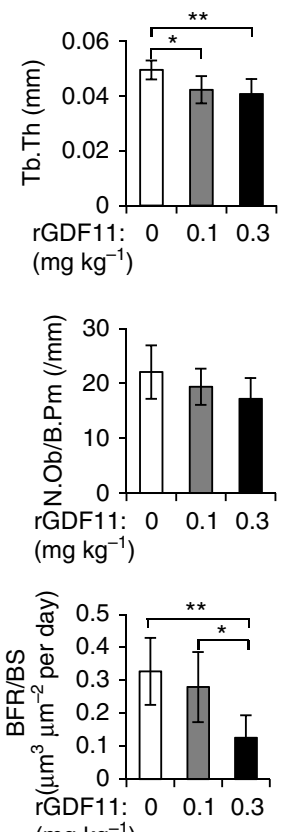

$\left(\mathrm{mg} \mathrm{kg}^{-1}\right)$

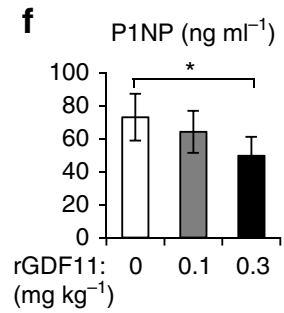

Figure 1 | GDF11 treatment leads to bone loss in young adult mice. (a) Representative images of microCT reconstruction of distal femurs. Scale bar $500 \mu \mathrm{m}$. (b) Von Kossa staining of undecalicified sections of femurs. Scale bar, $500 \mu \mathrm{m}$. (c) TRAP staining of femur sections from vehicle and rGDF11 treated mice. Scale bar, $50 \mu \mathrm{m}$. (d) Histomorphometric analysis of the metaphysis region of distal femurs. Results are shown as mean \pm s.d.; $n=6-10$; ${ }^{\star} P<0.05,{ }^{* \star} P<0.01$ and ${ }^{\star \star \star} P<0.001$ by analysis of variance (ANOVA) with Tukey's post hoc test. (e,f) The serum levels of CTX and P1NP. Results are shown as mean \pm s.d.; $n=6-10 ;{ }^{\star} P<0.05$ by ANOVA with Tukey's post hoc test.

Acp5 and Ctsk (Fig. 4b,c). Notably, a KEGG pathway analysis also indicated that GDF11 upregulated the expression of genes associated with TGF- $\beta$ pathway (Fig. $4 \mathrm{~d}, \mathrm{e}$ ). We also observed that rGDF11 was capable of activating the phosphorylation of Smad2/3 in mouse BMMs in vitro (Fig. $4 \mathrm{f}$, and Supplementary Fig. 4 for uncropped images). This is consistent with previous findings in myotubes and myocardium ${ }^{25,27}$. In addition, rGDF11 administration amplified the RANKL-induced expression of c-Fos (Fig. 3g). Immunohistochemical staining of the bone sections demonstrated that the numbers of $\mathrm{pSmad} 2 / 3$ and p-c-Fos-positive cells were increased after rGDF11 injections (Fig. 3h). A recent study showed that $S m a d 2 / 3$ cooperates with c-Fos to regulate the expression of $\mathrm{Nfatcl}$ during osteoclastogenesi $^{30}$. We observed increased expression of Nfatc1 upon rGDF11 treatment (Fig. 4b,c,h,i), so we performed a ChIP assay and found that rGDF11 significantly induced the recruitment of both $\operatorname{Smad} 2 / 3$ and c-Fos to the binding region of Nfatcl compared with RANKL treatment alone (Fig. 4j). We further investigated whether this effect is c-Fos dependent by performing loss of function experiments using c-Fos short interfering RNA (siRNA). Depletion of c-Fos eliminated the rGDF11 induced expression of Nfatcl (Fig. 4k), and completely abolished the triggered binding of Smad2/3 to Nfatc1 (Fig. 4l). These data, taken together, demonstrated that the effect of GDF11 on osteoclastogenesis is mediated by a Smad2/3 and c-Fos dependent increase in the transcription of $\mathrm{Nfatcl}$.
GDF11 inhibits osteoblast differentiation. We next investigated the effect of rGDF11 on osteoblast differentiation in vitro. BMSCs were cultured in osteogenic differentiation medium supplemented with or without rGDF11. The presence of rGDF11 $\left(100 \mathrm{ng} \mathrm{ml}^{-1}\right)$ significantly inhibited the osteogenic potential, as evidenced by alkaline phosphatase (ALP) staining and Alizarin Red S (ARS) staining (Fig. 5a). Quantitative analyses confirmed decreases in ALP activity (Fig. 5b) and calcium mineralization (Fig. 5c). Furthermore, quantitative PCR with reverse transcription (RT-PCR) revealed a reduction in messenger RNA expression of the master osteogenic transcription factors Runx2, as well as Osx (Osterix), Alp and Ocn (Osteocalcin) in rGDF11 treated BMSCs compared with controls (Fig. 5d). We also isolated primary calvarial osteoblasts for treatment with rGDF11. A similar, and sometimes an even more severe, inhibition of osteoblast differentiation was observed compared with the BMSCs (Fig. 5e-h). rGDF11 slightly increased the proliferation of both BMSCs and osteoblasts (Supplementary Fig. 2a,b).

We then determined whether GDF11 has a similar regulatory effect on the differentiation of osteoblasts via the TGF- $\beta$ pathway. Western blot showed that rGDF11 did stimulate the phosphorylation of Smad2/3 in primary osteoblasts (Fig. 5i). We found that rGDF11 inhibited the expression of Runx2 during osteogenic differentiation (Fig. 5d,h), which is in accordance with previous reports that the activation of $\mathrm{Smad} 3$ decreases the expression of Runx2 and represses its function by inducing 
a

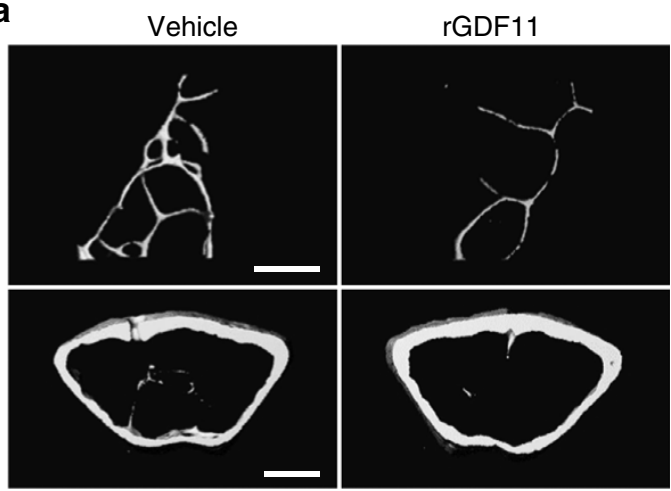

b

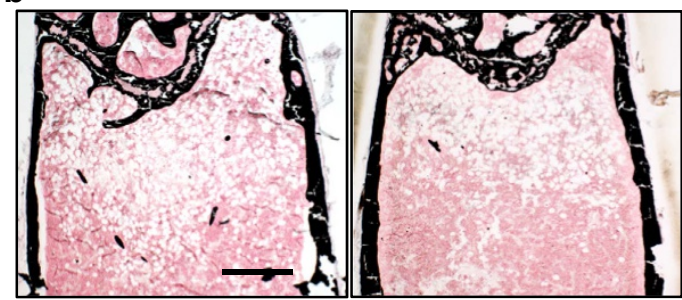

C

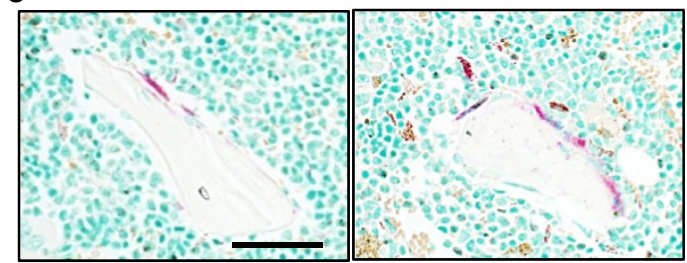

e

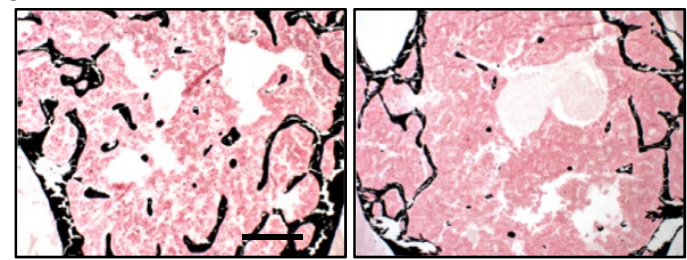

g

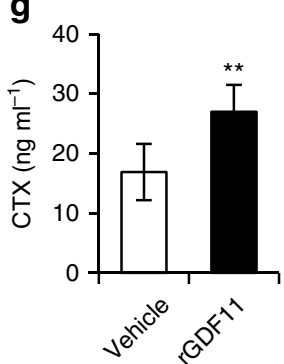

h

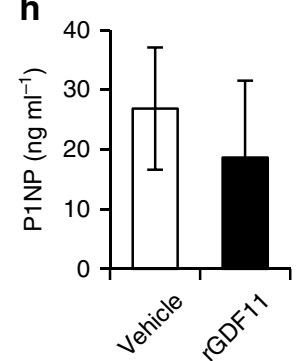

d
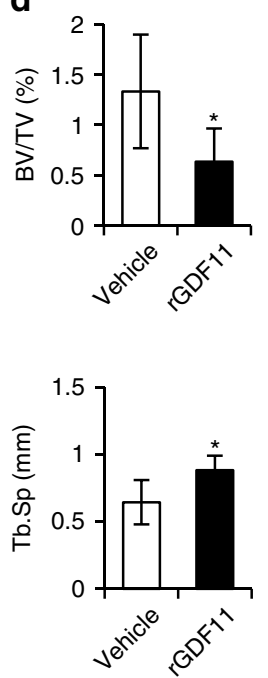

f
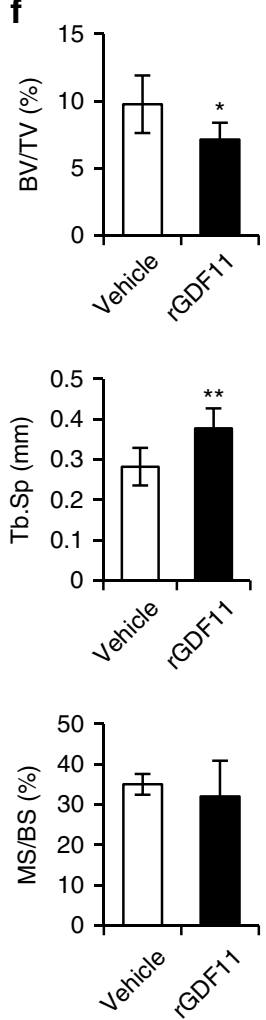
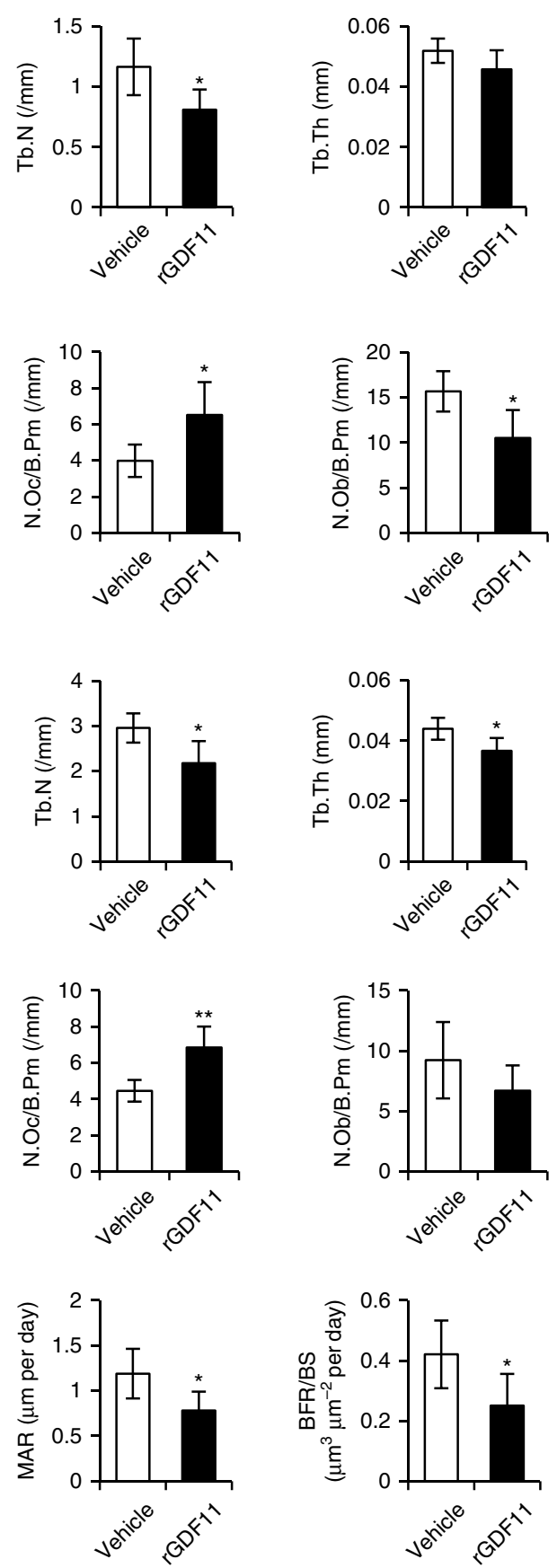

Figure 2 | GDF11 treatment leads to bone loss in aged mice. (a) Representative images of microCT reconstruction of distal femurs. Scale bar, $500 \mu \mathrm{m}$. (b) Von Kossa staining of undecalicified sections of femurs. Scale bar, $500 \mu \mathrm{m}$. (c) TRAP staining of femur sections from vehicle and rGDF11 treated mice. Scale bar, $50 \mu \mathrm{m}$. (d) Histomorphometric analysis of the metaphysis region of distal femurs. Results are shown as mean $\pm \mathrm{s}$.d.; $n=6$; ${ }^{\star} P<0.05$ by $t$ test. (e) Representative images of lumber 4 vertebrae stained with Von Kossa. Scale bar, $500 \mu \mathrm{m}$. (f) Histomorphometric analysis of the trabecular bone in vertebrae. Results are shown as mean \pm s.d.; $n=6 ;{ }^{*} P<0.05$ and ${ }^{\star \star} P<0.01$ by $t$ test. (g, $\left.\mathbf{h}\right)$ The serum levels of CTX and P1NP. Results are shown as mean \pm s.d.; $n=6$; ${ }^{\star \star} P<0.01$ by $t$ test.

histone $\mathrm{H} 4$ deacetylation at the osteocalcin promoter ${ }^{15,31}$. In addition, the ChIP assay demonstrated that rGDF11 significantly decreased the abundance of acetylated histone $\mathrm{H} 4$ (H4ac) at the Runx2 binding site of the osteocalcin promoter (Fig. 5j).
Activation of TGF- $\beta$ may counteract BMP signalling ${ }^{32,33}$, which is critical for osteoblast differentiation ${ }^{10}$. Therefore we evaluated the effect of rGDF11 on BMP signalling. Interestingly, we found that rGDF11 attenuated BMP2 (100 $\mathrm{ng} \mathrm{ml}^{-1}$, \#120-02C, PeproTech, Rocky Hill, NJ) or foetal bovine 


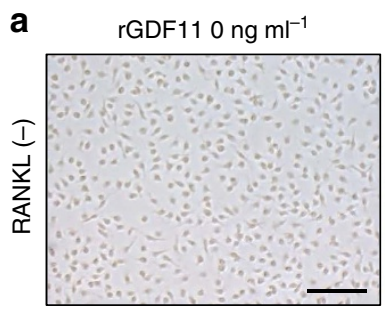

rGDF11 $50 \mathrm{ng} \mathrm{ml}^{-1}$
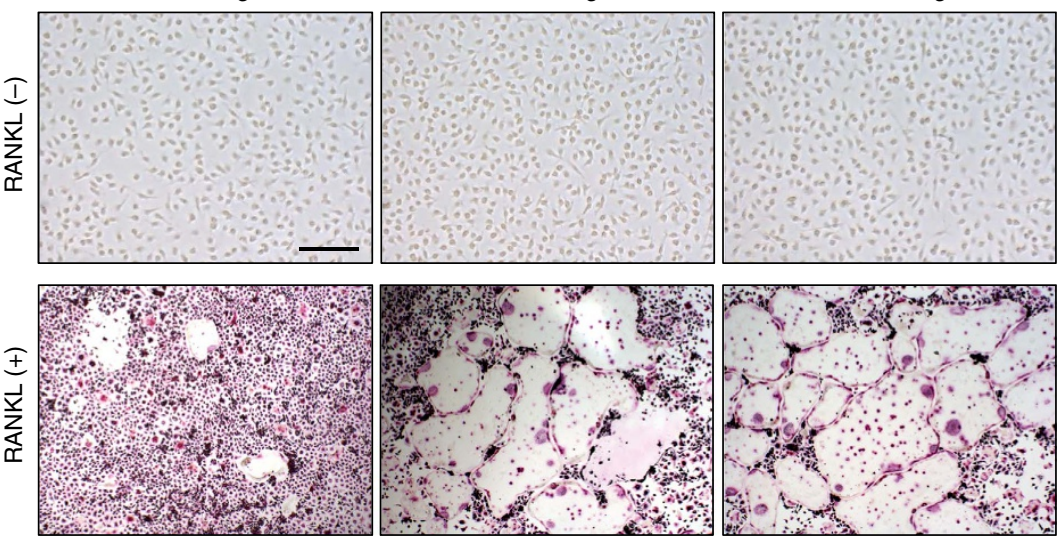

f
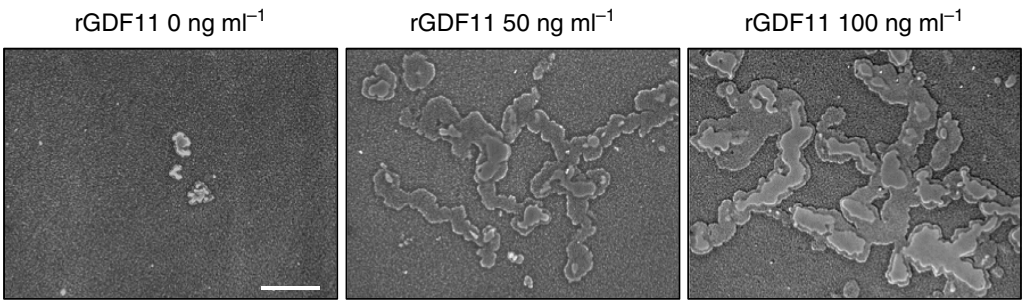

i

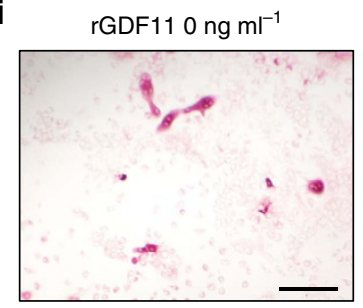

rGDF11 $50 \mathrm{ng} \mathrm{ml}^{-1}$

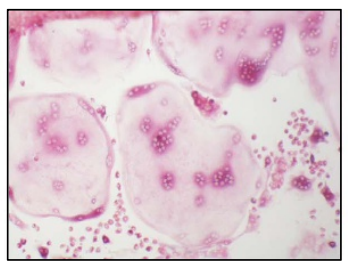

rGDF11 $100 \mathrm{ng} \mathrm{ml}^{-1}$

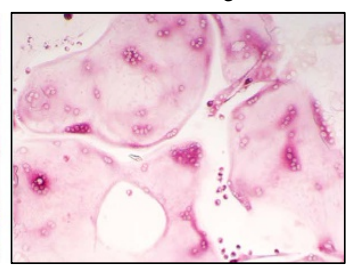

b

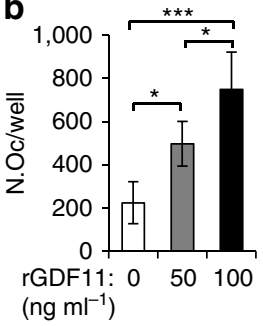

d

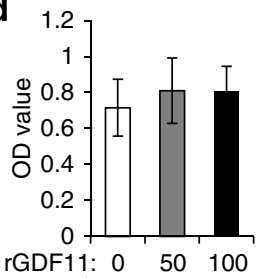

(ng ml ${ }^{-1}$ )

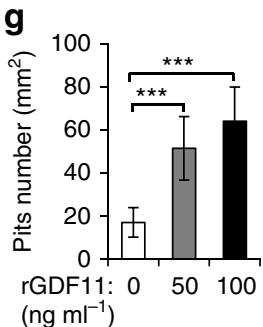

j

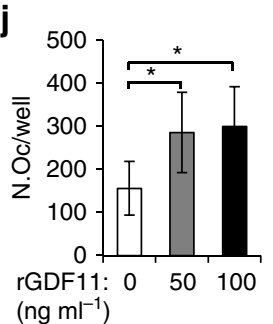

C
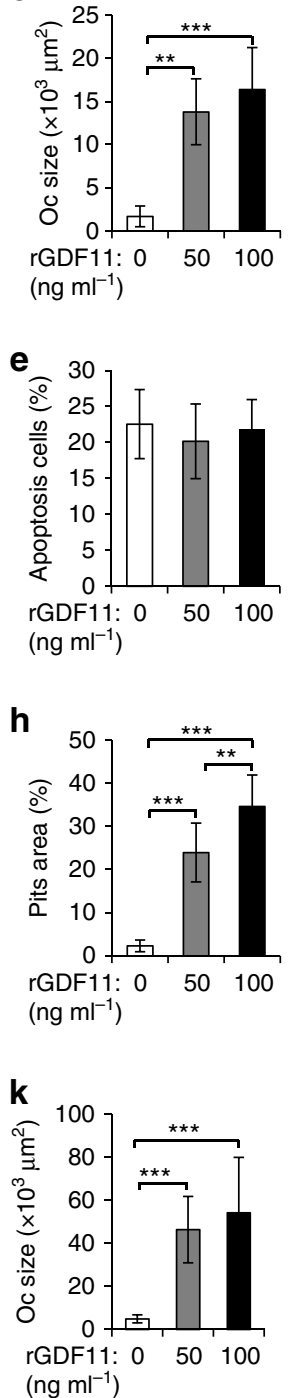

Figure 3 | GDF11 stimulates RANKL-induced osteoclastogenesis. (a) Representative TRAP staining of the BMMs after 4 days of osteoclast differentiation. $100 \mathrm{ng} \mathrm{ml}^{-1} \mathrm{M}$-CSF was present in all settings. Scale bar, $100 \mu \mathrm{m}$. (b,c) Osteoclast number and size. TRAP-positive cells with at least three nuclei were counted as osteoclasts. Results are shown as mean \pm s.d.; $n=9$; ${ }^{\star} P<0.05$, ${ }^{\star \star} P<0.01$ and ${ }^{\star \star \star} P<0.001$ by analysis of variance (ANOVA) with Tukey's post hoc test. (d,e) Cell proliferation and apoptosis assay. BMMs were cultured in the presence of $100 \mathrm{ng} \mathrm{ml}^{-1} \mathrm{M}^{-\mathrm{CSF}}$ and $50 \mathrm{ng} \mathrm{ml}-1$ RANKL with

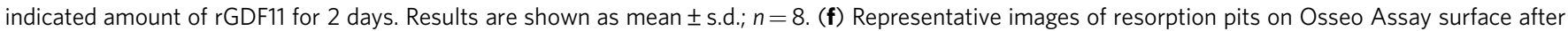
7 days of culture. Scale bar, $100 \mu \mathrm{m}$. (g,h) Resorption pits number and resorption area. Results are shown as mean $\pm \mathrm{s} . \mathrm{d}$.; $n=8 ;{ }^{\star \star} P<0.01$ and ${ }^{\star \star \star} P<0.001$ by ANOVA with Tukey's post hoc test. (i) Representative images of osteoclasts in co-cultures with osteoblasts in presence of $1,25(\mathrm{OH})_{2} \mathrm{D}_{3}$ and $\mathrm{PG} \mathrm{E}_{2}$ with indicated amount of rGDF11 for 5 days. Scale bar, $100 \mu \mathrm{m}$. (j,k) Osteoclast number and size for the co-cultures. Results are shown as mean \pm s.d.; $n=8$. ${ }^{\star} P<0.05$ and ${ }^{\star \star \star} P<0.001$ by ANOVA with Tukey's post hoc test.

serum (10\%) induced phosphorylation of Smad1/5 in osteoblasts (Fig. 5k,1).

GDF11 impairs the regeneration of bone defects. We further investigated the effect of rGDF11 in a bone regeneration model of femoral cortical bone defects induced by drill hole injury ${ }^{34}$. Young and aged mice were administered with rGDF11 $\left(0.3 \mathrm{mg} \mathrm{kg}^{-1}\right)$ or vehicle (saline) for 2 weeks before the surgeries, and rGDF11 was administered daily throughout the study. Mice were necropsied 2 weeks after surgeries. MicroCT and histological analyses consistently showed that the cortical gaps were almost completely bridged in both young and aged vehicle mice, while those of rGDF11 treated ones were only partially filled (Fig. 6a,b). The volume (BV/TV) and density (BMD) of the mineralized callus of rGDF11 group were significantly lower when compared with the vehicle group
(Fig. 6d). The osteoclast surfaces (Oc.S/BS) were largely elevated while osteoblast surfaces (Ob.S/BS) diminished (Fig. 6c,d). Immunohistochemical staining revealed more intensive pSmad2/3 signals in the rGDF11-treated group (Fig. 6e).

Next, we sought to determine whether GDF11 could have the same effect on bone regeneration of subcritical-sized calvarial defects. Two $1.0 \mathrm{~mm}$ bone defects were generated symmetrically across the sagittal suture. Only a small volume of newly formed bone was observed around the margin of this defect in the rGDF11 treated group (Fig. 6f,g). Moreover, the BV/TV and BMD were significantly lower than those in the vehicle group (Fig. 6h).

Inhibition of GDF11 prevents OVX-induced bone loss. Oestrogen deficiency in postmenopausal females leads to an imbalance between bone formation and bone resorption, which 

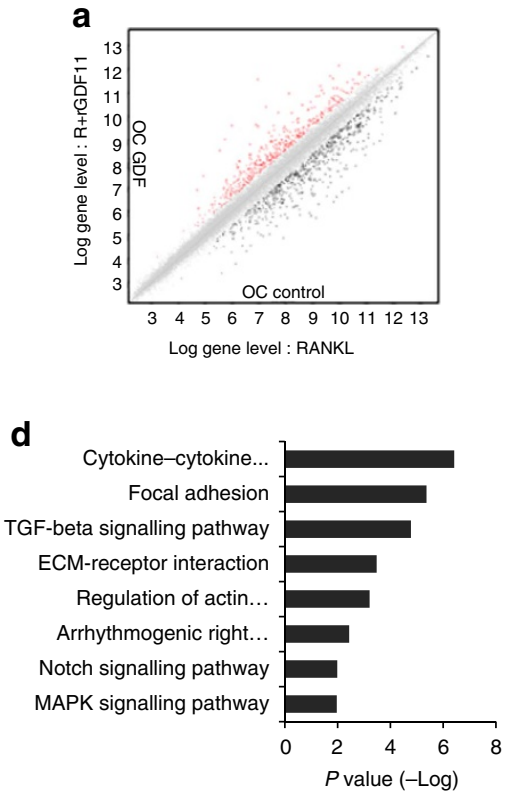

h
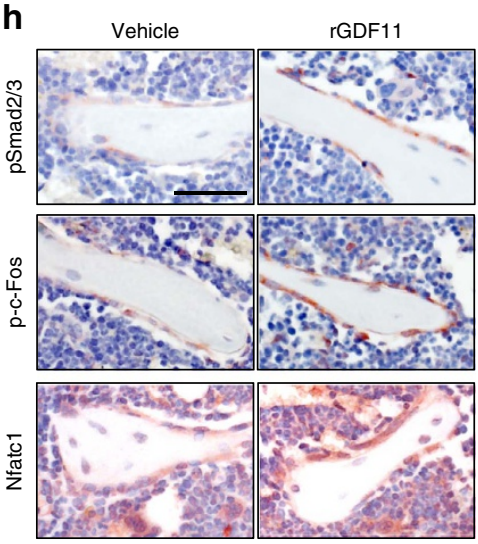

b
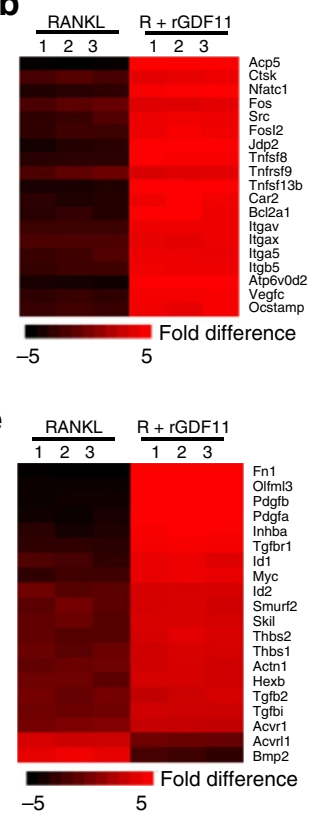

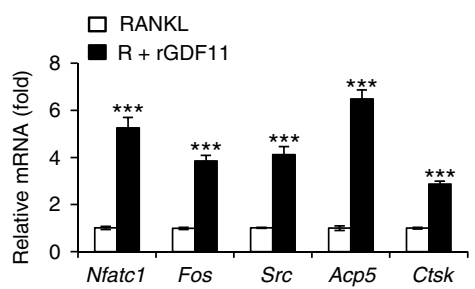

i

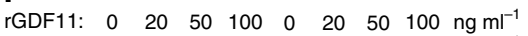
RANKL: $\begin{array}{cccccccccc}0 & 0 & 0 & 0 & 50 & 50 & 50 & 50 & \mathrm{ng} \mathrm{ml}^{-1}\end{array}$

Nfatc1

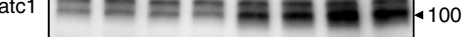

a-tub

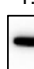

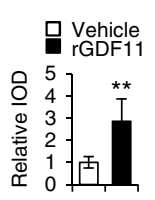

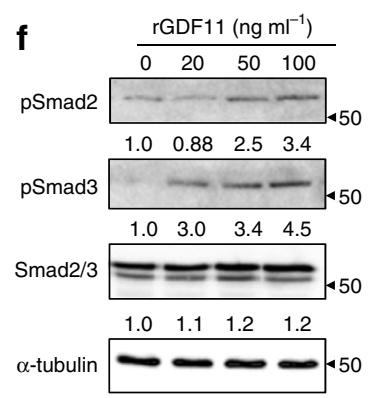

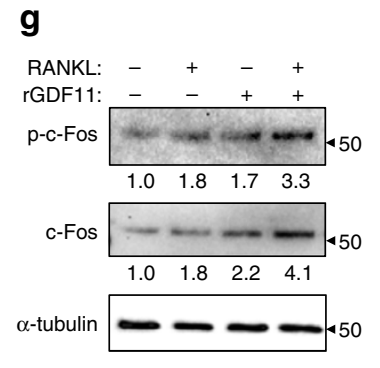

g

$\mathbf{k}$

Control siRnA C-Fos siRNA

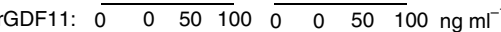
RANKL: $\quad 0 \quad 50 \quad 50 \quad 50 \quad 0 \quad 50 \quad 50 \quad 50 \quad \mathrm{ng} \mathrm{ml}^{-1}$ Nfatc1

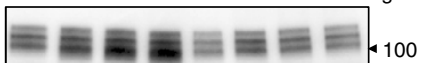

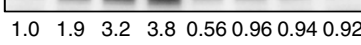
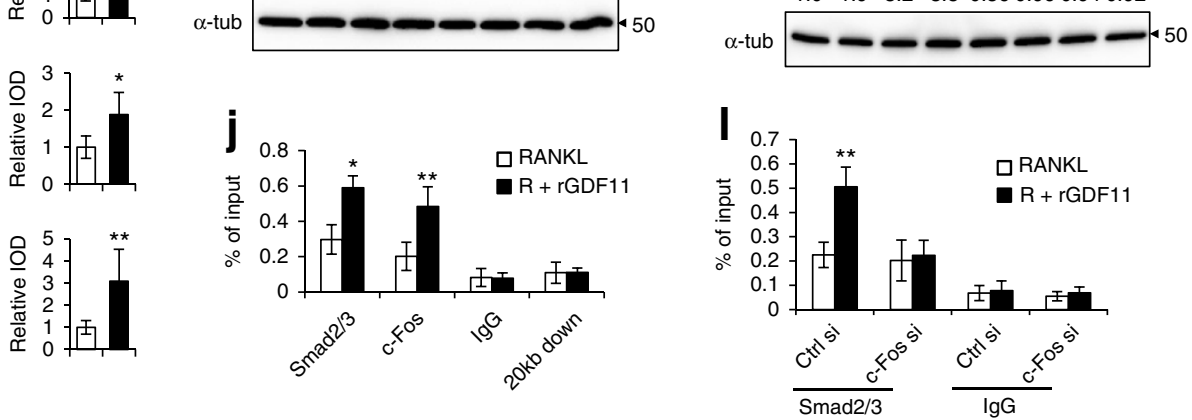

Figure 4 | GDF11 activates Smad2/3-dependent TGF- $\beta$ pathway. (a) Gene expression levels of the BMMs stimulated without or with 100 ng $\mathrm{ml}^{-1}$ rGDF11 for $24 \mathrm{~h}$ (three biological replicates per group). 467 genes (red) were upregulated and 679 genes (black) were downregulated. $100 \mathrm{ng} \mathrm{ml}^{-1} \mathrm{M}$-CSF was present in all settings. (b) Heatmap of the osteoclastogenesis associated genes. (c) Quantitative RT-PCR confirmed the increased expression of osteoclast key marker genes. Results are shown as mean \pm s.d.; $n=3$. ${ }^{\star \star \star} P<0.001$ by $t$ test. (d) KEGG pathway analysis indicated the altered function of TGF- $\beta$ pathway. (e) Heatmap of the TGF- $\beta$ pathway associated genes. (f) Western blot analysis indicated that rGDF11 stimulated the phosphorylation of Smad2/3 in BMMs in 30 min. (g) Western blot analysis demonstrated that rGDF11 amplified the RANKL-induced expression of c-Fos. BMMs were starved overnight and then treated for $4 \mathrm{~h}$. (h) Representative images of immunohistochemical staining. rGDF11 injections increased the phosphorylation of Smad2/3 and c-Fos, as well as the expression of Nfatc1 in vivo. Femurs were collected $\sim 2 \mathrm{~h}$ after the last injection of rGDF11. Scale bar, $50 \mu \mathrm{m}$. (i) Western blot analysis indicated that rGDF11 stimulated the RANKL-induced expression of Nfatc1. BMMs were treated for 2 days. (j) ChIP assay revealed that rGDF11 induced the co-occupancy of Smad2/3 and c-Fos to the binding region of Nfatc1. Results are shown as mean \pm s.d.; $n=3$. ${ }^{\star} P<0.05$ and ${ }^{\star \star} P<0.01$ by $t$ test. (k) Western blot analysis of Nfatc1. Depletion of c-Fos eliminated the rGDF11 induced expression of Nfatc1. (I) ChIP assay. Depletion of c-Fos abolished rGDF11 triggered binding of Smad2/3 to Nfatc1. Results are shown as mean \pm s.d.; $n=3 .{ }^{\star \star} P<0.01$ by $t$ test.

subsequently results in net bone loss and osteoporosis. Our results show that GDF11 plays a role in stimulating osteoclastogenesis and in inhibiting osteogenesis, so we hypothesized that inhibiting GDF11 could prevent oestrogen-deficiency-induced bone loss. To this end, sham and ovariectomized (OVX) mice were treated by intraperitoneal injections $(100 \mu \mathrm{g}$ per mouse per time, twice a week) of GDF11 antibody (Ab; MAB19581, clone \#743833, R\&D, Minneapolis, MN) starting 2 days after the OVX procedure and continuing for 6 weeks. GDF11 is highly related to myostatin (GDF8) and shares $90 \%$ homology in the mature active regions ${ }^{9}$. So we first performed western blot analysis to confirm that the batch of antibody we used in this experiment binds specifically to GDF11 without any cross-reaction with myostatin (Supplementary Fig. 3a,b). MicroCT analysis of the distal femur metaphysis revealed that the OVX procedure resulted in a nearly $50 \%$ bone loss in vehicle-treated mice (Fig. 7a,d). In contrast, the effect was significantly diminished in Ab-treated animals, where only a $21 \%$ bone loss occurred during the observation period (Fig. 7a,d). More importantly, the expected increases in osteoclast number (N. Oc/B.Pm) following OVX were significantly suppressed by GDF11 Ab administration (Fig. 7c,d). No differences were observed in the sham-operated mice treated 
a
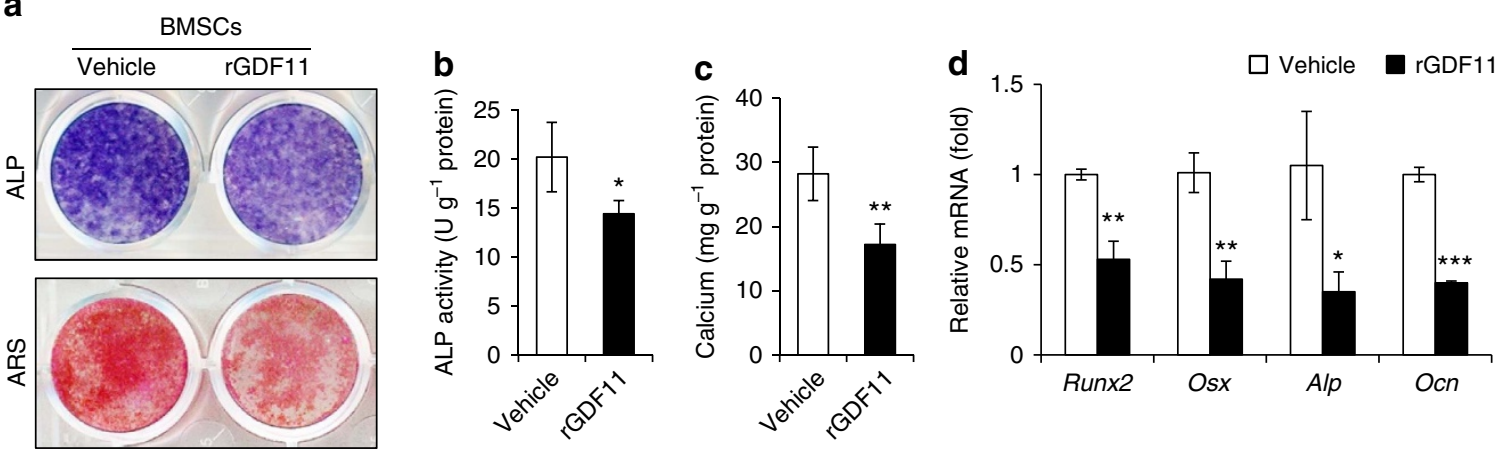

e
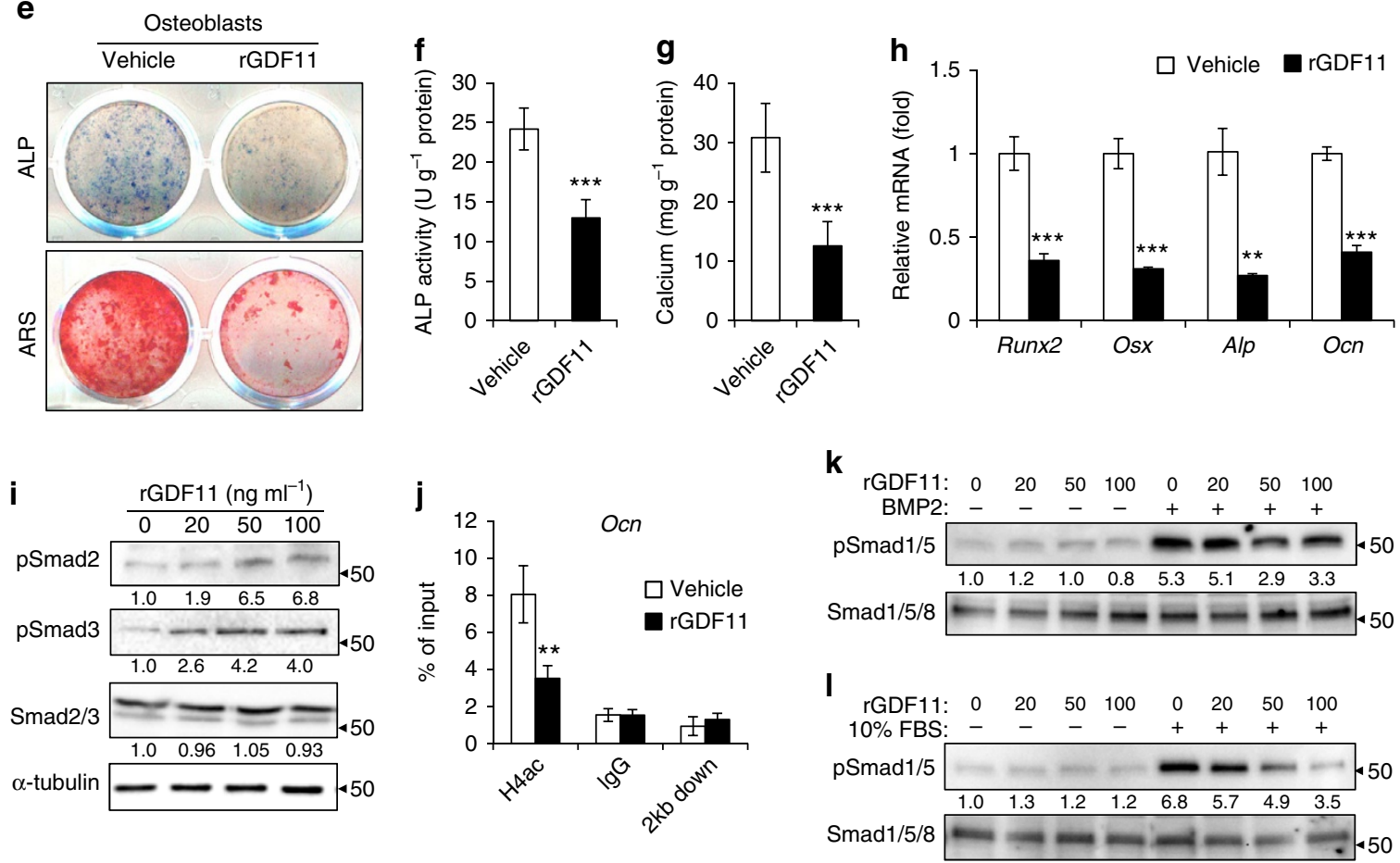

Figure 5 | GDF11 inhibits osteoblast differentiation. (a) Representative images of ALP staining and Alizarin Red S (ARS) staining of BMSCs.

$(\mathbf{b}, \mathbf{c})$ Quantitative analyses of the ALP activity and calcium mineralization in BMSCs. Results are shown as mean \pm s.d.; $n=5 ;{ }^{\star} P<0.05$ and ${ }^{\star \star} P<0.01$ by $t$ test. (d) Quantitative RT-PCR revealed reduced messenger RNA expression of Runx2, Osx, Alp and Ocn in rGDF11 treated BMSCs. Results are shown as mean \pm s.d.; $n=5 ;{ }^{\star} P<0.05,{ }^{\star \star} P<0.01$ and ${ }^{\star \star \star} P<0.001$ by $t$ test. (e) Representative images of ALP and ARS staining of primary calvarial osteoblasts. $(\mathbf{f}, \mathbf{g})$ Quantitative analyses of the ALP activity and calcium mineralization in osteoblasts. Results are shown as mean $\pm \mathrm{s}$.d.; $n=5 ;{ }^{\star \star \star} P<0.001$ by $t$ test. (h) Quantitative RT-PCR demonstrated that rGDF11 inhibited messenger RNA expression of Runx2, Osx, Alp and Ocn in osteoblasts. $n=3$. Results are shown as mean \pm s.d.; $n=5 ;{ }^{\star \star} P<0.01$ and ${ }^{\star \star \star} P<0.001$ by $t$ test. (i) Western blot indicated that rGDF11 stimulated the phosphorylation of Smad2/3 in osteoblasts. (j) ChIP assay revealed that rGDF11 reduced the abundance of acetylated histone $\mathrm{H} 4(\mathrm{H} 4 \mathrm{ac})$ on the promoter of Ocn. Results are shown as mean \pm s.d.; $n=3$; ${ }^{\star *} P<0.01$ by $t$ test. $(\mathbf{k}, \mathbf{I})$ Western blot analysis of $\mathrm{pSmad1} / 5$. The presence of $\mathrm{GGDF11}$ attenuated BMP2 or foetal bovine serum induced phosphorylation of Smad1/5. Osteoblasts were starved overnight and then treated for $30 \mathrm{~min}$.

with GDF11 Ab or vehicle (Fig. 7a-d). We also measured serum markers for bone turnover and found that the levels of CTX, a marker for bone resorption, were significantly reduced in GDF11 Ab-treated mice (Fig. 7e).

Inhibition of GDF11 ameliorates age-related osteoporosis. Based on these first observations, we further explored the therapeutic potential of GDF11 Ab for age-related osteoporosis. 18-month-old female mice were given intraperitoneal injections of GDF11 Ab $(100 \mu \mathrm{g}$ per mouse per time, twice a week) for 4 weeks. MicroCT and histomorphometric analyses of the L4 vertebrae showed that treatment with the GDF11 Ab significantly improved the trabecular bone volume (BV/TV; Fig. 7g,h,j). It also increased the trabecular number (Tb.N) and thickness (Tb.Th), while reducing the trabecular separation (Tb.Sp; Fig. 7j). Moreover, the osteoclast number (N.Oc/B.Pm) was significantly suppressed by GDF11 Ab administration (Fig. 7i,j). Taken together, our results suggest that GDF11 inhibition is a potential therapeutic approach for postmenopausal bone loss and age-related osteoporosis.

\section{Discussion}

Osteoporosis is an age-related disease that affects millions of people $e^{1,2}$. Aging populations around the world have fuelled continuing interest among biomedical researchers in finding antiaging factors ${ }^{35,36}$. It has recently been suggested that GDF11 is one of the long-sought rejuvenation agents ${ }^{22-24,37}$. Lee's group first reported that circulating levels of GDF11 decrease with age 
a

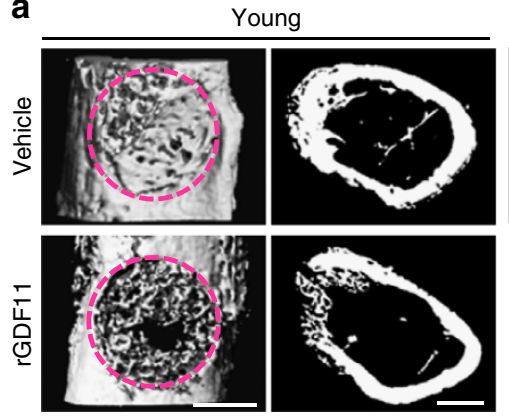

b

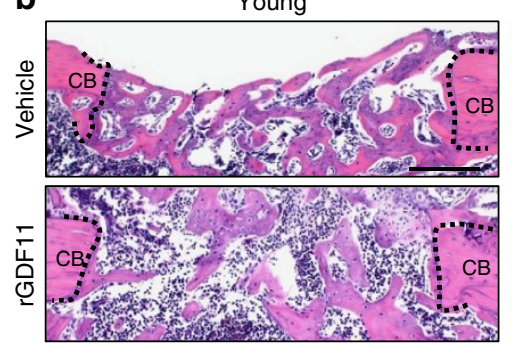

d

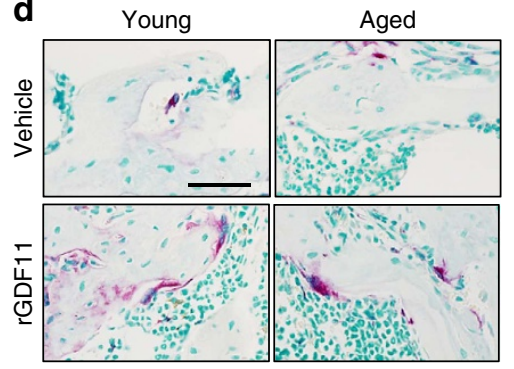

f
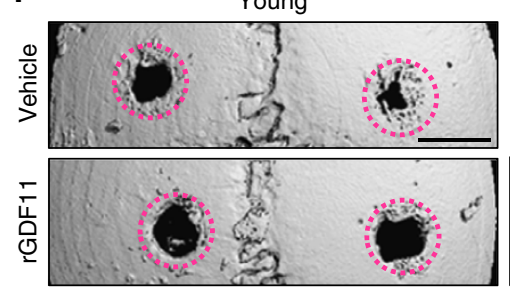

9
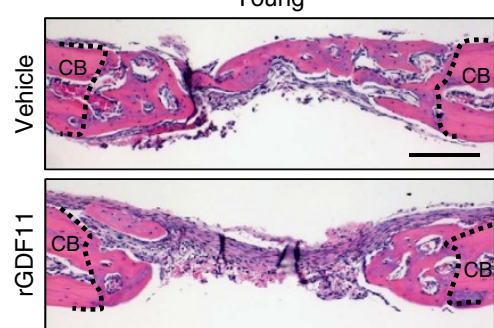
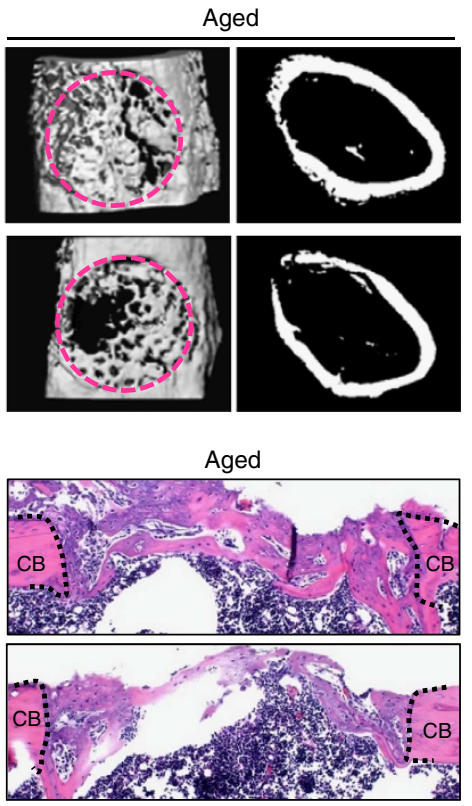
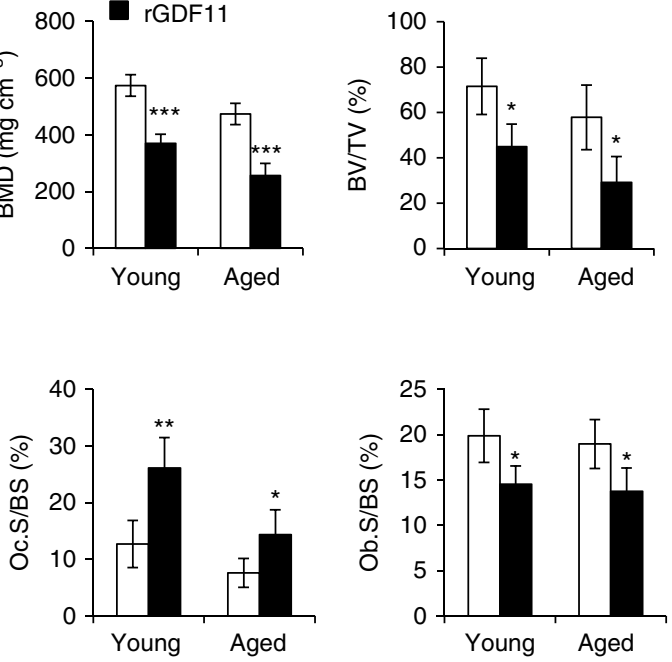
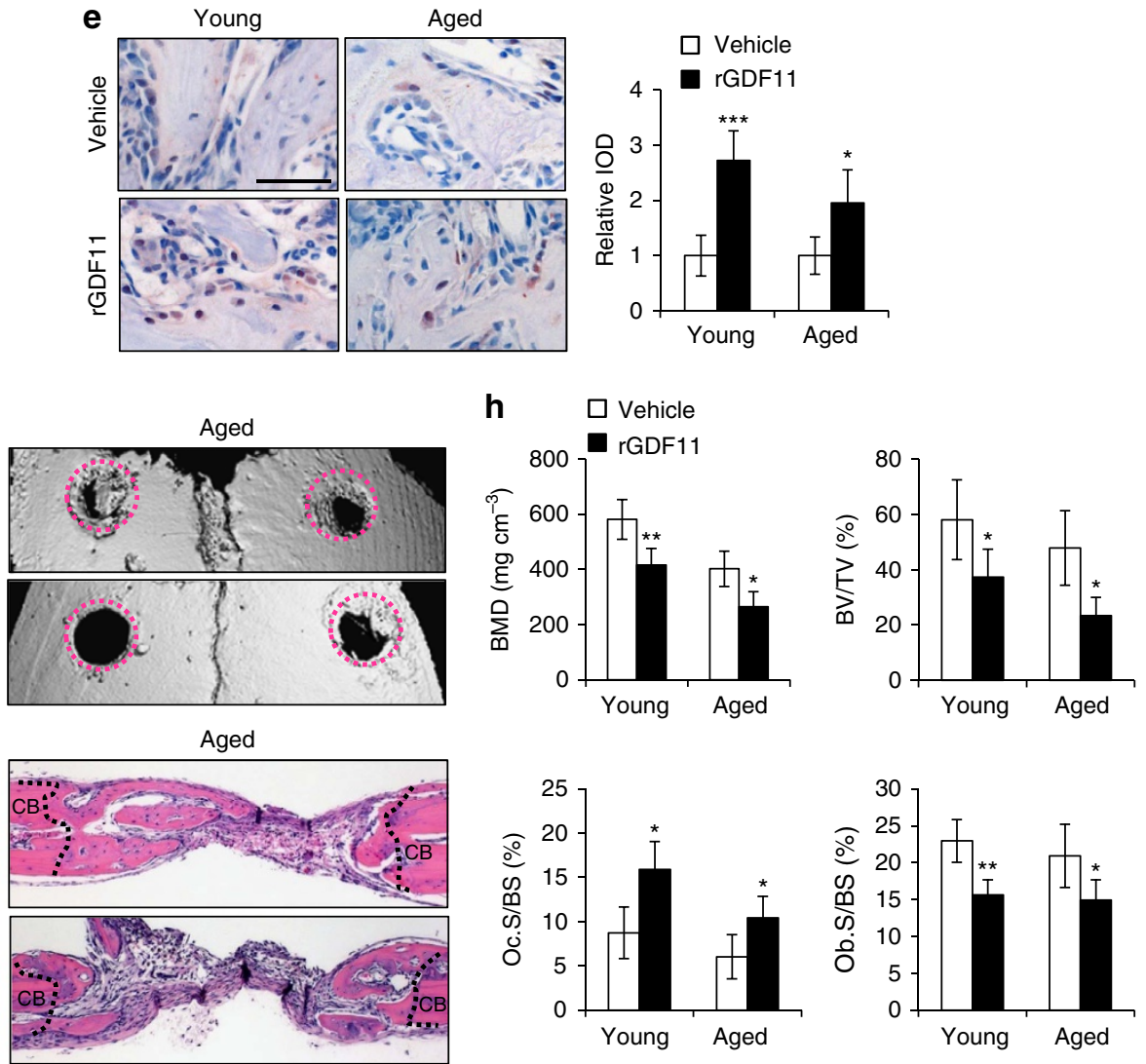

Figure 6 | GDF11 impairs regeneration of bone defects. (a) Representative images of microCT reconstruction of femoral cortical bone defects. The red dotted lines indicate the position of the original defect margin. Scale bar, $500 \mu \mathrm{m}$. (b) H\&E staining of femoral cortical bone defects. The black dotted lines indicate the position of the original defect margin. Abbreviation: $C B$, cortical bone. Scale bar, $200 \mu \mathrm{m}$. (c) Bone mineral density (BMD) and

histomorphometric analysis of the regenerated bone in femoral cortical gaps. Results are shown as mean $\pm s$.d. The mean values of the bilateral defects of each mouse were counted as individual data points $(n=5)$. ${ }^{\star} P<0.05$, ${ }^{\star \star} P<0.01$ and ${ }^{\star \star \star} P<0.001$ by $t$ test. (d) Representative images of TRAP staining of the regenerated bone in femoral cortical gaps. Scale bar, $50 \mu \mathrm{m}$. (e) Representative images and quantification of immunohistochemical staining of pSmad2/3. rGDF11 injections increased the phosphorylation of Smad2/3. Scale bar, $50 \mu \mathrm{m}$. (f) MicroCT reconstruction of the calvarial defects. The red dotted lines indicate the position of the original defect margin. Scale bar, $1 \mathrm{~mm}$. (g) H\&E staining of the calvarial defects. The dotted lines indicate the position of the original defect margin. CB, cortical bone. Scale bar, $200 \mu \mathrm{m}$. (h) Bone mineral density (BMD) and histomorphometric analysis of the regenerated bone in calvarial defects. Results are shown as mean \pm s.d. The mean values of the bilateral defects of each mouse were counted as individual data points $(n=5)$. ${ }^{\star} P<0.05$ and ${ }^{\star \star} P<0.01$ by $t$ test. 
a

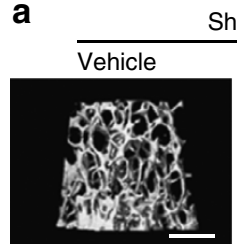

Sham

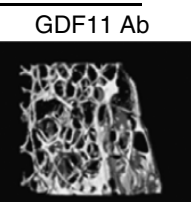

b
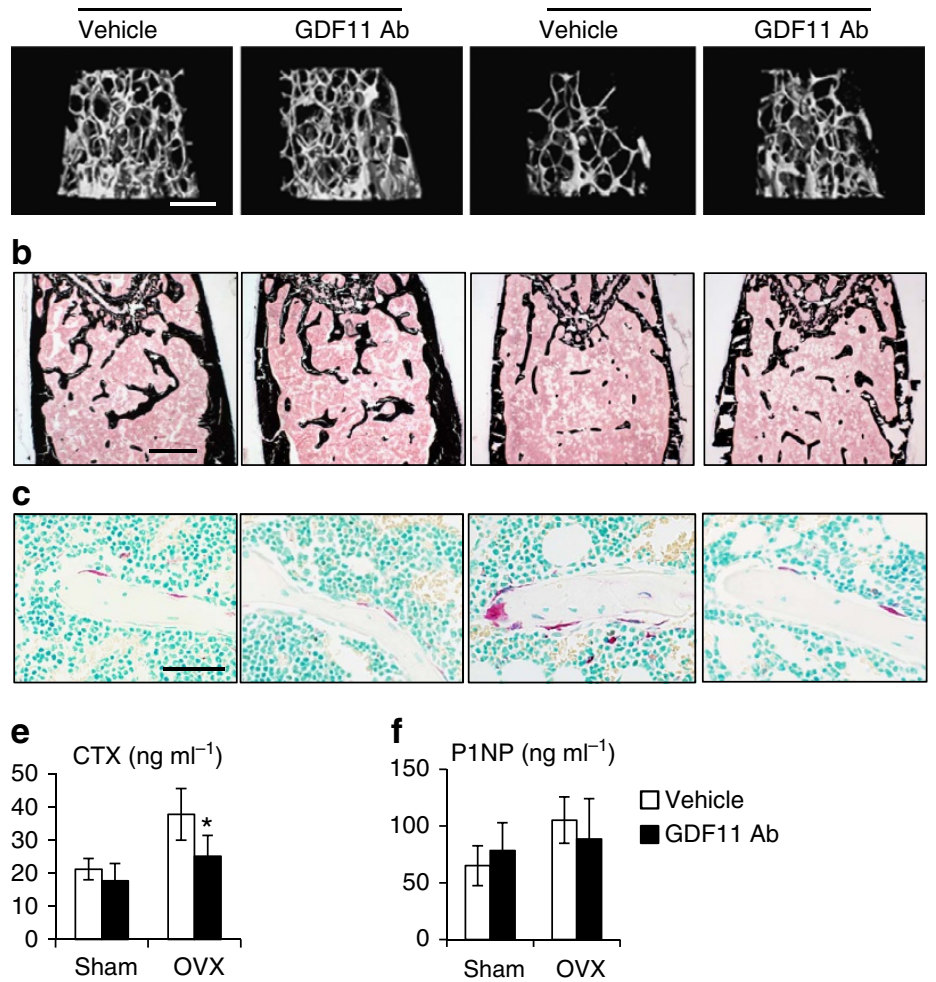

d
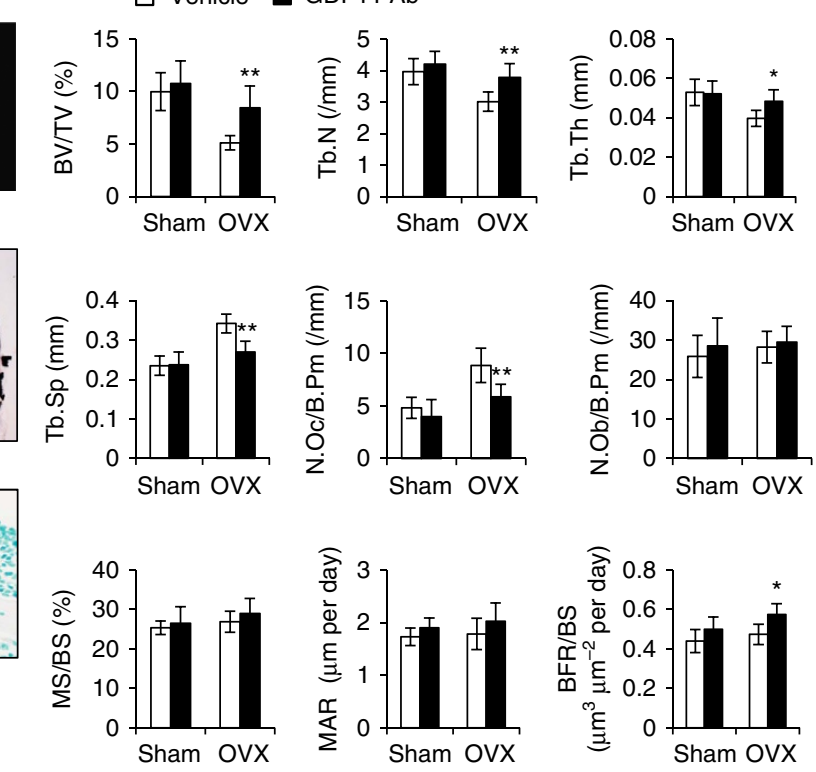

\section{g}
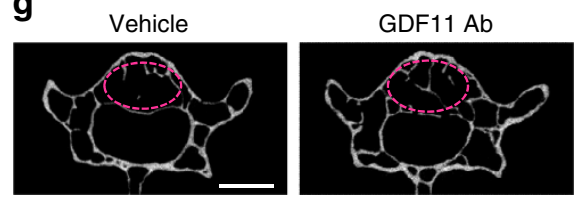

h

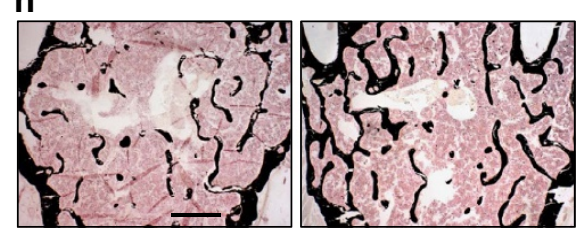

i

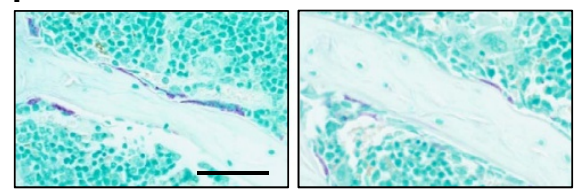

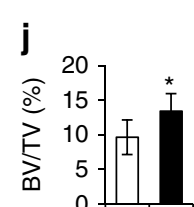

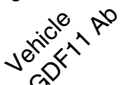
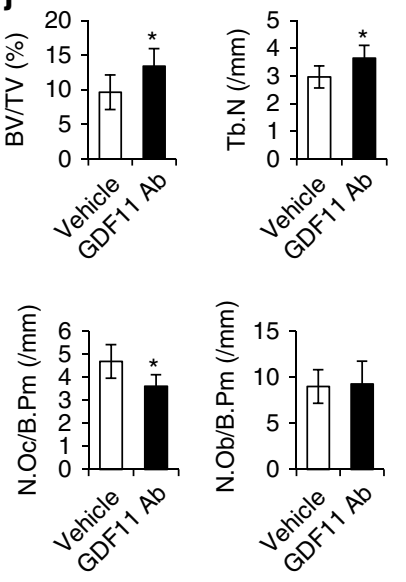
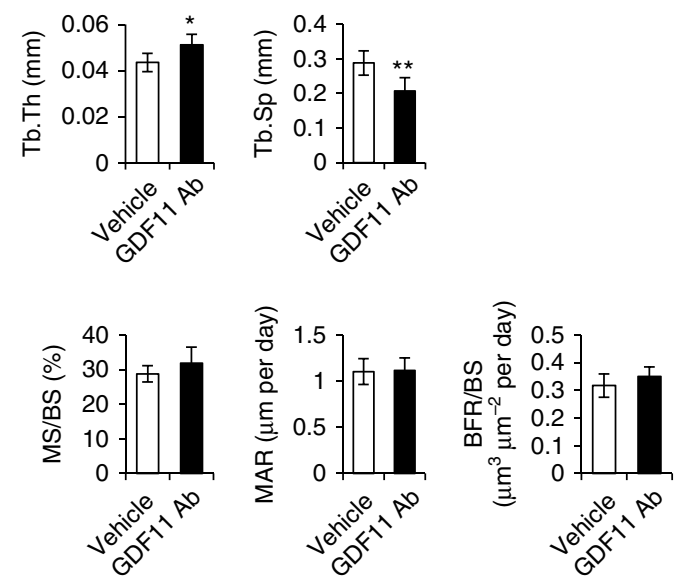

Figure 7 | Inhibition of GDF11 prevents bone loss. (a) MicroCT reconstruction of trabecular bone from distal femurs. Scale bar, $500 \mu \mathrm{m}$. (b) Von Kossa staining of undecalicified sections of femurs. Scale bar, $500 \mu \mathrm{m}$. (c) TRAP staining of femur sections. Scale bar, $50 \mu \mathrm{m}$. (d) Histomorphometric analysis of the metaphysis region of distal femurs. Results are shown as mean \pm s.d.; $n=6 ;{ }^{\star} P<0.05$ and ${ }^{\star \star} P<0.01$ by $t$ test. (e,f) The serum levels of $C T X$ and P1NP. Results are shown as mean \pm s.d.; $n=6$; ${ }^{\star} P<0.05$ by $t$ test. (g) Representative images of microCT scanning of lumber 4 vertebrae. The red circles indicate the region of interest (ROI). Scale bar, $1 \mathrm{~mm}$. (h) Von Kossa staining of undecalicified sections of lumber 4 vertebrae. Scale bar, $500 \mu \mathrm{m}$. (i) TRAP staining of vertebrae sections. Scale bar, $50 \mu \mathrm{m}$. (j) Histomorphometric analysis of the trabecular bone in lumber four vertebrae. Results are shown as mean \pm s.d.; $n=6 ;{ }^{*} P<0.05$ and ${ }^{* \star} P<0.01$ by $t$ test.

and restoration of more youthful levels of systemic GDF11 appears to reverse age-related cardiac hypertrophy ${ }^{22}$. Later related studies suggested that injection of rGDF11 rejuvenates skeletal, muscle and brain function in aged mice ${ }^{23,24}$. A clinical study also suggested that higher GDF11/8 levels are associated with a lower risk of cardiovascular events and death in patients with stable ischaemic heart disease ${ }^{38}$. However, these observations were recently challenged ${ }^{26-29,39-43}$. Egerman et al. ${ }^{27}$ demonstrated that circulating GDF11 increases with age and systematic administration of rGDF11 actually inhibits muscle regeneration. Others also reported that GDF11 lacks an antihypertrophic effect in vitro and restoring GDF11 in old mice has no effect on cardiac structure or function ${ }^{29}$. In this study, we injected both young and aged mice with rGDF11, and did not observe any significant change in the pool of BMSCs in bone marrow or their ability for colony formation. Most recently, 
Schafer et al. ${ }^{26}$ showed that in humans, GDF11 levels do not change with age but are associated with comorbidity and frailty. In addition, Lee's group ${ }^{25}$ verified their previous reports that serum GDF11/8 levels decline with age in multiple mammalian species. Their results indicated that the increase in GDF11 levels, as reported by Egerman et al..$^{27}$, is due to a cross-reaction of the GDF8/GDF11 antibody (Abcam) with immunoglobulin. This issue might be resolved with experiments performed parallel using the R\&D GDF11 antibody. Others ${ }^{27,29}$ and we have verified that this antibody specifically detects GDF11 without cross-reaction with myostatin.

In this study, we first reported that rGDF11 treatment decreases bone mass that results from the uncoupling of bone resorption with bone formation. Previously, Li et al. ${ }^{44}$ generated transgenic mice that over-express the GDF11 propeptide under the control of a bone-specific regulatory element. The GDF11 propeptide forms a latent complex with GDF11 to antagonize GDF11 activity ${ }^{45}$, so this mouse might be considered as a model for repression of GDF11 function. Indeed, these transgenic mice exhibited an abnormal transformation of vertebral formation ${ }^{44}$. More importantly, these mice also showed a significant increase in bone mass ${ }^{19}$, which supports our finding that GDF11 induces bone loss. Recently, Zhang et al. ${ }^{46}$ reported a controversial finding that GDF11 induces osteoblast differentiation. However, we could not reproduce their results using rGDF11 from the same manufacturer and the same methods as they described. The conflicting findings may be caused by different batches of GDF11 from the manufacturer. Cells from different sources may be another possibility, but Zhang et al. did not describe the details of cell collection.

It should be noted that we could not fully exclude an indirect effect on bone mass caused by the systematic application of rGDF11. Recently, accumulating evidence suggests that GDF11 treatment may negatively affect the skeletal muscle ${ }^{27,40,42}$, although deletion of Gdf11 specifically in skeletal muscle does not affect muscle size, fibre number or fibre type ${ }^{47}$. Given the role of muscle to bone crosstalk ${ }^{48-50}$, GDF11 treatment may also regulate bone mass indirectly by affecting skeletal muscle. In addition, we still lack direct genetic evidence for the role of GDF11 in postnatal bone remodelling. Gdf11 knockout mice die shortly after birth and none of the currently available knockout or transgenic mouse models could avoid the effect of losing GDF11 during prenatal development ${ }^{17,44,51,52}$. A better approach is to generate the inducible knockout or overexpression mouse models for future studies.

GDF11 is highly related to myostatin (GDF8) with $90 \%$ homology in the mature active regions ${ }^{9}$. These two proteins also have redundant functions in regulating skeletal patterning in mice ${ }^{47}$. Here we show that GDF11 inhibits osteoblast differentiation and stimulates RANKL-induced osteoclastogenesis, which is also similar to the functions of myostatin. Previous studies showed that loss of myostatin increases osteogenic differentiation in BMSCs and that myostatin-null mice exhibit improved bone mass and bone mineral density ${ }^{53-55}$. Recently, Dankbar et al. ${ }^{16}$ demonstrated that myostatin directly regulates osteoclast differentiation and its inhibition reduces inflammatory joint destruction in mice. The effects of GDF11 and myostatin on osteoclasts also appear similar as both stimulate RANKL-mediated osteoclastogenesis through the Smad2/3dependent TGF- $\beta$ pathway. GDF11 rapidly induces the phosphorylation of Smad2/3 and c-Fos, both in vitro and in vivo, and increases the transcription of $\mathrm{Nfatcl}$.

In this study, we demonstrate that GDF11 is a previously unrecognized regulator of bone remodelling. The bone loss induced by rGDF11 injections likely represents a pharmacological effect of rGDF11, given their low endogenous levels in mice.
However, we did show that blocking of endogenous GDF11 function prevents oestrogen-deficiency-induced bone loss and age-related osteoporosis, indicating GDF11 does have effects on bone metabolism physiologically. Some novel treatment strategies for osteoporosis have been developed previously ${ }^{2}$. A prominent example is denosumab, which is a monoclonal antibody for RANKL that aims to inhibit excessive bone resorption. We show that GDF11 is a strong stimulator of RANKL-induced osteoclast formation. Therefore, GDF11 inhibition might be a potential new approach for osteoporosis treatment, especially in patients with high levels of GDF11.

In summary, we show that GDF11 treatment leads to bone loss in both young and aged mice. We further show that GDF11 stimulates RANKL-induced osteoclast formation in vitro. This effect is mainly through increased phosphorylation of $\operatorname{Smad} 2 / 3$ and c-Fos, which subsequently stimulates the transcription of Nfatc1. GDF11 also inhibits osteoblast differentiation by Smad2/3-dependent repression of Runx2. In addition, rGDF11 treatment impairs bone regeneration in both young and aged mice, and blocking GDF11 function prevents oestrogendeficiency-induced bone loss and ameliorates age-related osteoporosis. Our data demonstrate that GDF11 is a critical regulator of bone remodelling, and suggest that GDF11 may be a potential target for treatment of osteoporosis.

\section{Methods}

Animals. Female C57BL mice were obtained from the Experimental Animal Center of Sichuan University and housed in pathogen-free facilities under a 12-h light and 12-h dark cycle. All protocols were approved by the Subcommittee on Research and Animal Care (SRAC) of Sichuan University.

MicroCT analysis. MicroCT analysis was performed according to recent guidelines $^{56}$ using a Skyscan 1176 microCT imaging system (Skyscan, Kontich, Belgium) with a spatial resolution of $12 \mu \mathrm{m}$ (X-ray source $50 \mathrm{kV} / 455 \mathrm{kA}$; exposure time $0.265 \mathrm{~s}$; magnification $\times 15 ; 1 \mathrm{~mm}$ filter applied). Volumetric reconstructions and analyses were performed using built-in software NRecon 1.6 and CTAn 1.8, respectively. For the analysis of bone regeneration, the volume of interest was defined as a cylindrical area covering the initial bone defect. Bone volume (BV/TV, \%) was calculated within the delimited volume of interest.

Bone histology and histomorphometry. Processing of undecalcified bone specimens and cancellous bone histomorphometry were performed as described previously ${ }^{57}$. Femurs and vertebrae were fixed in $10 \%$ buffered formalin overnight and then stored in $70 \%$ ethanol at $4{ }^{\circ} \mathrm{C}$ before being processed and embedded in methylmethacrylate. Five- $\mu$ m-thick sections were prepared using a Leica RM2235 microtome and were stained by the von Kossa/nuclear fast red method. Histomorphometric measurements in the distal femur and L4 vertebrae were made using OsteoMeasure software (OsteoMetrics, Decatur, GA). All histomorphometric parameters were calculated and expressed according to the suggestions made by the ASBMR nomenclature committee ${ }^{58}$.

ELISA. Serum concentrations of CTX and P1NP were measured using commercial ELISA kits from IDS (Fountain Hills, AZ). Blood was collected 4 weeks after rGDF11 treatment or 2 weeks after GDF11 Ab treatment by puncturing the cheek pouch of animals. Before blood collection, the mice were fasted for $4 \mathrm{~h}$.

Cell culture. We cultured the cells in minimum essential medium $\alpha$ ( $\alpha$-MEM) supplemented with $10 \%$ foetal bovine serum, 100 units $\mathrm{ml}^{-1}$ penicillin, $100 \mathrm{~g} \mathrm{ml}^{-1}$ streptomycin and $10 \mathrm{mM}$ HEPES (all from Gibco). Mouse BMMs were cultured as follows. Tibiae of 6-9-week-old mice were aseptically removed and the bone marrow cells were flushed out. The cells were suspended and cultured in the medium containing $100 \mathrm{ng} \mathrm{ml}^{-1}$ macrophage colony-stimulating factor (M-CSF) (416-ML/CF, R\&D, Minneapolis, MN) overnight at $37^{\circ} \mathrm{C}$. The untouched cells were collected and further cultured with $100 \mathrm{ng} \mathrm{ml}^{-1} \mathrm{M}$-CSF for 2 days to obtain BMMs. The resultant BMMs were then differentiated into osteoclasts in the presence of $100 \mathrm{ng} \mathrm{ml}^{-1} \mathrm{M}$-CSF and $50 \mathrm{ng} \mathrm{ml}^{-1} \mathrm{RANKL}$ (462-TR/CF, R\&D, Minneapolis, MN) with or without rGDF11 (\#120-11, PeproTech, Rocky Hill, NJ) for 4 days. Subsequently, cells were fixed and stained using TRAP for evaluation. We counted TRAP-positive cells with at least three nuclei as osteoclasts. For the resorption assay, BMMs were seeded on an Osseo Assay surface (Corning) and differentiated into osteoclasts. After 7 days, the cells were removed by incubation 
with $10 \%$ bleach solution for $10 \mathrm{~min}$, and the resorption pits were visualized by phase microscopy.

BMSCs were isolated from 9-week-old mice by flushing the bone marrow of tibiae and femurs ${ }^{59}$. Primary osteoblasts were isolated from the calvariae of 3 -day-old mice as described previously ${ }^{60}$. Briefly, calvariae were dissected aseptically, and sequentially digested with $1 \mathrm{mg} \mathrm{ml}^{-1}$ collagenase solution containing collagenase types I and II in 1:3 ratio (Worthington, Newark, NJ). Osteoblast enriched fractions (fraction 3 to 6) were collected and cultured for around 7 days until confluence. Osteoblast differentiation was induced by treating cells with medium containing $50 \mu \mathrm{M}$ ascorbic acid, $10 \mathrm{mM} \beta$-glycerophosphate and $10 \mathrm{nM}$ dexamethasone (all from Sigma). For co-culture assay, BMMs were seeded on the confluent osteoblast layer and cultured for 5 days in presence of $10 \mathrm{nM}$ 1,25-dihydroxyvitamin $\mathrm{D}_{3}\left(1,25(\mathrm{OH})_{2} \mathrm{D}_{3}\right.$, Sigma) and $1 \mu \mathrm{M}$ prostaglandin $\mathrm{E}_{2}\left(\mathrm{PGE}_{2}\right.$, Sigma) with or without $100 \mathrm{ng} \mathrm{ml}^{-1} \mathrm{rGDF} 11$.

Cell sorting and colony formation assay. We collected the bone marrow cells from the tibiae of rGDF11- or vehicle-treated animals by flushing. Cells were incubated in the red blood cell lysis buffer for $5 \mathrm{~min}$ at room temperature. The sorting of BMSCs were performed as described ${ }^{61}$. Briefly, the cell aliquots were incubated with phycoerythrin (PE)-, fluorescein isothiocyanate (FITC)-, peridinin chlorophyll protein (Per CP)-, and allophycocyanin (APC)-conjugated antibodies against mouse CD29, Sca-1, CD11b and CD45 (BioLegend, San Diego, CA) at $4{ }^{\circ} \mathrm{C}$ for $30 \mathrm{~min}$. Cell sorting was performed on a fluorescence-activated cell sorting (FACS) Aria model (BD Biosciences), and analysis was performed with fluorescence-activated cell sorting DIVE software version 6.1.3 (BD Biosciences).

The sorted Sca- $1^{+} \mathrm{CD} 29^{+} \mathrm{CD} 45^{-} \mathrm{CD} 11 \mathrm{~b}^{-}$cells were collected and plated into 6-well plates (1,000 cells per well). After $4 \mathrm{~h}$ of adhesion, unattached cells were removed. Medium was changed every 3 days, and cultures were fixed and stained with $0.5 \mathrm{mg} \mathrm{ml}^{-1}$ of crystal violet on day 10 . Colonies containing 50 or more cells were counted.

siRNA-mediated knockdown. Mouse c-Fos and control small interfere RNAs (siRNAs) were purchased from Santa Cruz (Dallas, Texas). Each siRNA consists of pools with three to five target-specific 19-25-nucleotide siRNAs designed to knockdown target gene expression. For siRNA-mediated knockdown, mouse BMMs were transfected using Lipofectamine RNAimax (Invitrogen) following the manufacturer's instruction.

Proliferation and apoptosis assay. The proliferation of BMMs, BMSCs and osteoblasts was determined using Cell Proliferation Reagent WST-1 (Roche, Indianapolis, IN). Briefly, $10 \mu \mathrm{l}$ of reagent was added to each well, including three wells containing only medium for background subtraction. After incubation at $37^{\circ} \mathrm{C}$ for $1 \mathrm{~h}$, the absorbance at $450 \mathrm{~nm}$ was measured using a microplate reader (Varioskan Flash, ThermoScientific). The apoptosis assay of BMMs was performed using an in situ Cell Death Detection kit (Roche) according to the manufacturer's instruction.

Microarray analysis and quantitative RT-PCR. Expression levels of messenger RNA were measured by microarray analysis of mouse BMMs $24 \mathrm{~h}$ after stimulation in the presence of $100 \mathrm{ng} \mathrm{ml}^{-1} \mathrm{M}$-CSF and $50 \mathrm{ng} \mathrm{ml}^{-1}$ RANKL with or without $100 \mathrm{ng} \mathrm{ml}^{-1}$ rGDF11. Total RNA was isolated using an RNeasy kit (Qiagen) according to the manufacturer's protocol. Microarray experiments were performed in triplicate using the Affymetrix Mouse Gene 1.0 ST Array. The robust multichip average method was used to normalize the gene expression raw data. We calculated the gene expression levels using Affymetrix Expression Console and Transcriptome Analysis Console 3.0 software. The threshold for differentially regulated transcripts was set as the fold change of 1.5 with a $P$ value of $<0.01$

For quantitative RT-PCR, cDNA was prepared from $2 \mu \mathrm{g}$ RNA using a QuantiTec reverse transcription kit (Qiagen) and analysed with SYBR GreenMaster Mix (SABiosciences) in ABI7500 real-time PCR system (Applied Biosystems, Foster City, CA). The primers designed for each targeted gene are listed in Supplementary table 1 . Relative expression was calculated using a $2^{-\Delta \Delta \mathrm{Ct}}$ method by normalizing with Gapdh housekeeping gene expression and presented as fold increase relative to control.

Western blot. Cells were lysed in RIPA buffer (Pierce, Rockford, IL) on ice. The samples were denatured at $95^{\circ} \mathrm{C}$ for $5 \mathrm{~min}$ in sample buffer containing $2 \%$ SDS and $1 \% 2$-mercaptoethanol, separated on 10 or $15 \%$ SDS-polyacrylamide gels, and transferred to polyvinylidene difluoride membranes by a semi-dry transfer apparatus (Bio-Rad, Hercules, CA). For native condition, rGDF11 or Myostatin (\#120-00, PeproTech, Rocky Hill, NJ) were added into the loading buffer $(62.5 \mathrm{mM}$ Tris-HCl, $\mathrm{pH}$ 6.8; 40\% glycerol; and 0.01\% Bromophenol Blue), and electrophoresed on $4-15 \%$ Mini-PROTEAN TGX gels (Bio-Rad, Hercules, CA) in a running buffer containing $25 \mathrm{mM}$ Tris Base and $192 \mathrm{mM}$ glycine at $4{ }^{\circ} \mathrm{C}$. The proteins were then transferred to polyvinylidene difluoride membranes at $4^{\circ} \mathrm{C}$ by a wet transfer apparatus (Bio-Rad, Hercules, CA). The membranes were blotted with $5 \%$ milk for $1 \mathrm{~h}$ and then incubated with primary antibodies overnight. The following antibodies were used: mouse anti-GDF11 $\left(0.5 \mu \mathrm{g} \mathrm{ml}{ }^{-1}\right.$, MAB19581, clone \#743833, R\&D), rabbit anti-phospho-Smad2 (1:1,000, MA5-15122 l, ThermoFisher), rabbit anti-phospho-Smad3 (1:1,000, \#9520, Cell Signaling), rabbit anti-phospho-Smad2/3 (1:1,000, \#8828, Cell Signaling), rabbit anti-Smad2/3 (1:1,000, \#8685, Cell Signaling), rabbit anti-phospho-c-Fos (1:1,000, \#5348, Cell Signaling), rabbit anti-c-Fos (1:1,000, \#2250, Cell Signaling), mouse anti-NFATc1 (1:2,000, MA3-024, ThermoFisher), rabbit anti-phospho-Smad1/5 (1:1,000, \#9516, Cell Signaling), and rabbit anti-Smad1/5/8 (1:1,000, SC-6031-R, Santa Cruz). The immunocomplexes were incubated with horseradish peroxidase conjugated anti-rabbit or anti-mouse IgG (Jackson ImmunoResearch, West Grove, PA) and visualized with SuperSignal reagents (Pierce, Rockford, IL). The ImageJ software was used for densitometric analyses of the blots, and the quantification results were normalized to the loading control.

Chromatin Immunoprecipitation (ChIP) assay. The antibodies for ChIP were as follows: acetylated histone H4 (H4ac, \#39926, Active Motif), Smad2/3 (\#8685, Cell Signaling) and c-Fos (\#2250, Cell Signaling). ChIP assays were performed using a SimpleChIP Assay Kit (Cell Signaling Technology) according to the manufacturer's protocol. Briefly, cells were incubated in $5 \mathrm{mM}$ dimethyl $3,3^{\prime}$-dithiobispropionimidate-HCl (DTBP; Pierce, Rockford, IL) for $10 \mathrm{~min}$ at room temperature, rinsed with $100 \mathrm{mM}$ Tris- $\mathrm{HCl}$ buffered saline $(\mathrm{pH} 8.0)$ and crosslinked with $1 \%$ formaldehyde in PBS for $10 \mathrm{~min}$ at $37^{\circ} \mathrm{C}$. Precipitated DNA samples were quantified with real-time PCR. Data are presented as the percentage of input DNA. The primers are listed in Supplementary table 1.

Immunohistochemical staining. The dissected femurs were fixed with $10 \%$ buffered formalin overnight and decalcified with 15\% EDTA in a cold room for 2 weeks before processing for paraffin sections. Sections were treated in sodium citrate buffer ( $10 \mathrm{mM}$ Sodium citrate, $0.05 \%$ Tween $20, \mathrm{pH} 6.0)$ at $90^{\circ} \mathrm{C}$ for $20 \mathrm{~min}$ for antigen retrieval and incubated with rabbit anti-phospho-Smad2/3 (1:100, \#8828, Cell Signaling; or sc-11769, Santa Cruz), rabbit anti-phospho-c-Fos (1:200, \#5348, Cell Signaling) or mouse anti-NFATc1 (1:100, MA3-024, ThermoFisher) antibodies overnight at $4{ }^{\circ} \mathrm{C}$. The DAKO Envision + HRP (AEC) system was used to detect primary antibodies. We quantified the integral optical density (IOD) of positive staining using Image Pro Plus 6.0 (MediaCybernetics) and normalized by stained area.

ALP and mineralization assay. BMSCs and primary calvarial osteoblasts were grown in osteogenic differentiation medium supplemented with or without $100 \mathrm{ng} \mathrm{ml}^{-1}$ rGDF11. On day 7, cells were fixed with $3.7 \%$ formaldehyde and incubated with a staining solution of $0.25 \%$ naphthol AS-BI phosphate and $0.75 \%$ Fast Blue BB dissolved in 0.1 M Tris buffer ( $\mathrm{pH}$ 9.3). ALP activity was also quantified using a commercial kit according to the manufacturer's protocol (Cell Biolab, San Diego, CA)

For mineralization assays, cells were cultured in differentiation medium for 2 weeks, fixed with $3.7 \%$ formaldehyde and stained with $1 \%$ Alizarin red S (pH 4.2 Sigma-Aldrich) for $10 \mathrm{~min}$. Mineralized bone nodules stained with alizarin red were destained with $10 \%$ cetylpyridinium chloride in $10 \mathrm{mM}$ sodium phosphate (pH 7.0), and the calcium concentration was determined by absorbance measurements at $562 \mathrm{~nm}$ using a standard calcium curve in the same solution.

Surgeries. Young (9 week old) and aged (18 month old) female mice were anaesthetized with intraperitoneal injection of a combination of ketamine $\left(100 \mathrm{mg} \mathrm{kg}^{-1}\right)$ and xylazine $\left(10 \mathrm{mg} \mathrm{kg}^{-1}\right)$. In addition, buprenorphine $\left(0.05 \mathrm{mg} \mathrm{kg}^{-1}\right)$ was given for perioperative analgesia to minimize suffering and pain. Femoral cortical bone defects were created as described ${ }^{34,62,63}$. Briefly, the medial surfaces of the bilateral femurs (distal portion) were exposed by blunt dissection of the quadriceps after skin incision. A $1.0 \mathrm{~mm}$ hole was generated using a round bur (Komet, Germany) operating at 10,000 r.p.m. under saline irrigation.

Subcritical-sized cranial defects were created following the published surgical protocols ${ }^{62,64}$. After careful exposure of the flat surface of the cranium via skin incision, two $1.0 \mathrm{~mm}$ symmetrical full-thickness bone defects were generated across the sagittal suture using a cylindrical low-speed carbide bur (Komet, Germany). Periosteum was closed using interrupted 5-0 Monocryl sutures before skin closure.

For bilateral ovariectomy (OVX), the ovaries of 9-week-old animals were approached through a midline dorsal skin incision and exposed by cutting through the muscle layer. Both ovaries were removed after ligating the uterine horn, and the incision of the muscle was sutured with 6-0 silk. The skin was closed with surgical clips. Sham surgery included anaesthesia, midline dorsal incision, exteriorizing and returning of the ovary, and closure of the wound. Antibody treatment started 2 days after the surgery.

Statistical analysis. All values are expressed as mean \pm s.d. Statistically significant differences were evaluated by two-tailed Student's $t$ test for comparison between two groups or by one-way analysis of variance followed by the Tukey's test for multiple comparisons. A $P$ value of $<0.05$ was considered to be statistically significant. 
Data availability. Microarray data that support the findings of this study have been deposited in NCBI GEO with the primary accession code GSE84609. The authors declare that all other data supporting the findings of this study are available within the article and its Supplementary information files.

\section{References}

1. NIH Consensus Development Panel on Osteoporosis Prevention, Diagnosis, and Therapy. Osteoporosis prevention, diagnosis, and therapy. JAMA 285, 785-795 (2001).

2. Rachner, T. D., Khosla, S. \& Hofbauer, L. C. Osteoporosis: now and the future. Lancet 377, 1276-1287 (2011).

3. Crane, J. L. \& Cao, X. Bone marrow mesenchymal stem cells and TGF-beta signaling in bone remodeling. J. Clin. Invest. 124, 466-472 (2014).

4. Zaidi, M. Skeletal remodeling in health and disease. Nat. Med. 13, 791-801 (2007).

5. Manolagas, S. C. \& Parfitt, A. M. What old means to bone. Trends Endocrinol. Metab. 21, 369-374 (2010).

6. Raisz, L. G. Pathogenesis of osteoporosis: concepts, conflicts, and prospects. J. Clin. Invest. 115, 3318-3325 (2005).

7. Raggatt, L. J. \& Partridge, N. C. Cellular and molecular mechanisms of bone remodeling. J. Biol. Chem. 285, 25103-25108 (2010).

8. Yu, B. et al. Wnt4 signaling prevents skeletal aging and inflammation by inhibiting nuclear factor-kappaB. Nat. Med. 20, 1009-1017 (2014).

9. Nakashima, M., Toyono, T., Akamine, A. \& Joyner, A. Expression of growth/ differentiation factor 11, a new member of the BMP/TGFbeta superfamily during mouse embryogenesis. Mech. Dev. 80, 185-189 (1999).

10. Rahman, M. S., Akhtar, N., Jamil, H. M., Banik, R. S. \& Asaduzzaman, S. M. TGF-beta/BMP signaling and other molecular events: regulation of osteoblastogenesis and bone formation. Bone Res. 3, 15005 (2015).

11. Quinn, J. M. et al. Transforming growth factor beta affects osteoclast differentiation via direct and indirect actions. J. Bone Miner. Res. 16, 1787-1794 (2001).

12. Sells Galvin, R. J., Gatlin, C. L., Horn, J. W. \& Fuson, T. R. TGF-beta enhances osteoclast differentiation in hematopoietic cell cultures stimulated with RANKL and M-CSF. Biochem. Biophys. Res. Commun. 265, 233-239 (1999).

13. Bonewald, L. F. \& Dallas, S. L. Role of active and latent transforming growth factor beta in bone formation. J. Cell. Biochem. 55, 350-357 (1994).

14. Breen, E. C. et al. TGF beta alters growth and differentiation related gene expression in proliferating osteoblasts in vitro, preventing development of the mature bone phenotype. J. Cell. Physiol. 160, 323-335 (1994).

15. Alliston, T., Choy, L., Ducy, P., Karsenty, G. \& Derynck, R. TGF-beta-induced repression of CBFA1 by Smad3 decreases cbfal and osteocalcin expression and inhibits osteoblast differentiation. EMBO J. 20, 2254-2272 (2001).

16. Dankbar, B. et al. Myostatin is a direct regulator of osteoclast differentiation and its inhibition reduces inflammatory joint destruction in mice. Nat. Med. 21, 1085-1090 (2015).

17. McPherron, A. C., Lawler, A. M. \& Lee, S. J. Regulation of anterior/posterior patterning of the axial skeleton by growth/differentiation factor 11. Nat. Genet. 22, 260-264 (1999).

18. Oh, S. P. et al. Activin type IIA and IIB receptors mediate Gdf11 signaling in axial vertebral patterning. Genes Dev. 16, 2749-2754 (2002).

19. Li, Z. et al. Transgenic overexpression of bone morphogenetic protein 11 propeptide in skeleton enhances bone formation. Biochem. Biophys. Res. Commun. 416, 289-292 (2011).

20. Nakashima, M., Mizunuma, K., Murakami, T. \& Akamine, A. Induction of dental pulp stem cell differentiation into odontoblasts by electroporationmediated gene delivery of growth/differentiation factor 11 (Gdf11). Gene Ther. 9, 814-818 (2002)

21. Nakashima, M. et al. Stimulation of reparative dentin formation by ex vivo gene therapy using dental pulp stem cells electrotransfected with growth/ differentiation factor 11 (Gdf11). Hum. Gene Ther. 15, 1045-1053 (2004).

22. Loffredo, F. S. et al. Growth differentiation factor 11 is a circulating factor that reverses age-related cardiac hypertrophy. Cell 153, 828-839 (2013).

23. Katsimpardi, L. et al. Vascular and neurogenic rejuvenation of the aging mouse brain by young systemic factors. Science 344, 630-634 (2014).

24. Sinha, M. et al. Restoring systemic GDF11 levels reverses age-related dysfunction in mouse skeletal muscle. Science 344, 649-652 (2014).

25. Poggioli, T. et al. Circulating growth differentiation factor $11 / 8$ levels decline with age. Circ. Res. 118, 29-37 (2016)

26. Schafer, M. J. et al. Quantification of GDF11 and myostatin in human aging and cardiovascular disease. Cell Metab. 23, 1207-1215 (2016).

27. Egerman, M. A. et al. GDF11 increases with age and inhibits skeletal muscle regeneration. Cell Metab. 22, 164-174 (2015).

28. Glass, D. J. Elevated GDF11 is a risk factor for age-related frailty and disease in humans. Cell Metab. 24, 7-8 (2016).

29. Smith, S. C. et al. GDF11 does not rescue aging-related pathological hypertrophy. Circ. Res. 117, 926-932 (2015).
30. Omata, Y. et al. Genomewide comprehensive analysis reveals critical cooperation between Smad and c-Fos in RANKL-induced osteoclastogenesis. J. Bone Miner. Res. 30, 869-877 (2015).

31. Kang, J. S., Alliston, T., Delston, R. \& Derynck, R. Repression of Runx2 function by TGF-beta through recruitment of class II histone deacetylases by Smad3. EMBO J. 24, 2543-2555 (2005).

32. Ehnert, S. et al. Transforming growth factor betal inhibits bone morphogenic protein (BMP)-2 and BMP-7 signaling via upregulation of Ski-related novel protein $\mathrm{N}(\mathrm{SnoN})$ : possible mechanism for the failure of BMP therapy? BMC Med. 10, 101 (2012).

33. Gronroos, E. et al. Transforming growth factor beta inhibits bone morphogenetic protein-induced transcription through novel phosphorylated Smad1/5-Smad3 complexes. Mol. Cell Biol. 32, 2904-2916 (2012).

34. Hayashi, M. et al. Osteoprotection by semaphorin 3A. Nature 485, 69-74 (2012).

35. Scudellari, M. Ageing research: blood to blood. Nature 517, 426-429 (2015).

36. Sun, H. \& Wang, Y. The elusive philosopher's stone in young blood. Circ. Res. 117, 906-908 (2015).

37. Kaiser, J. 'Rejuvenation factor' in blood turns back the clock in old mice. Science 344, 570-571 (2014).

38. Olson, K. A. et al. Association of growth differentiation factor 11/8, putative anti-ageing factor, with cardiovascular outcomes and overall mortality in humans: analysis of the Heart and Soul and HUNT3 cohorts. Eur. Heart J. 36, 3426-3434 (2015).

39. Brun, C. E. \& Rudnicki, M. A. GDF11 and the mythical fountain of youth. Cell Metab. 22, 54-56 (2015)

40. Rodgers, B. D. \& Eldridge, J. A. Reduced circulating GDF11 is unlikely responsible for age-dependent changes in mouse heart, muscle, and brain. Endocrinology 156, 3885-3888 (2015).

41. Harper, S. C. et al. Is growth differentiation factor 11 a realistic therapeutic for aging-dependent muscle defects? Circ. Res. 118, 1143-1150 (2016).

42. Hinken, A. C. et al. Lack of evidence for GDF11 as a rejuvenator of aged skeletal muscle satellite cells. Aging Cell 15, 582-584 (2016).

43. Rodgers, B. D. The immateriality of circulating GDF11. Circ. Res. 118, 1472-1474 (2016).

44. Li, Z., Kawasumi, M., Zhao, B., Moisyadi, S. \& Yang, J. Transgenic over-expression of growth differentiation factor 11 propeptide in skeleton results in transformation of the seventh cervical vertebra into a thoracic vertebra. Mol. Reprod. Dev. 77, 990-997 (2010).

45. Ge, G., Hopkins, D. R., Ho, W. B. \& Greenspan, D. S. GDF11 forms a bone morphogenetic protein 1-activated latent complex that can modulate nerve growth factor-induced differentiation of PC12 cells. Mol. Cell Biol. 25, 5846-5858 (2005).

46. Zhang, Y. et al. Growth differentiation factor 11 is a protective factor for osteoblastogenesis by targeting PPARgamma. Gene 557, 209-214 (2015).

47. McPherron, A. C., Huynh, T. V. \& Lee, S. J. Redundancy of myostatin and growth/differentiation factor 11 function. BMC Dev. Biol. 9, 24 (2009).

48. Brotto, M. \& Bonewald, L. Bone and muscle: interactions beyond mechanical. Bone 80, 109-114 (2015)

49. Brotto, M. \& Johnson, M. L. Endocrine crosstalk between muscle and bone Curr. Osteoporos. Rep. 12, 135-141 (2014).

50. Cianferotti, L. \& Brandi, M. L. Muscle-bone interactions: basic and clinical aspects. Endocrine 45, 165-177 (2014).

51. Gamer, L. W., Cox, K. A., Small, C. \& Rosen, V. Gdf11 is a negative regulator of chondrogenesis and myogenesis in the developing chick limb. Dev. Biol. 229, 407-420 (2001).

52. Esquela, A. F. \& Lee, S. J. Regulation of metanephric kidney development by growth/differentiation factor 11. Dev. Biol. 257, 356-370 (2003).

53. Hamrick, M. W. et al. Loss of myostatin (GDF8) function increases osteogenic differentiation of bone marrow-derived mesenchymal stem cells but the osteogenic effect is ablated with unloading. Bone 40, 1544-1553 (2007).

54. Hamrick, M. W. Increased bone mineral density in the femora of GDF8 knockout mice. Anat. Rec. A Discov. Mol. Cell Evol. Biol. 272, 388-391 (2003).

55. Hamrick, M. W., Pennington, C. \& Byron, C. D. Bone architecture and disc degeneration in the lumbar spine of mice lacking GDF-8 (myostatin). J. Orthop. Res. 21, 1025-1032 (2003).

56. Bouxsein, M. L. et al. Guidelines for assessment of bone microstructure in rodents using micro-computed tomography. J. Bone Miner. Res. 25, 1468-1486 (2010).

57. Yuan, Q. et al. Increased osteopontin contributes to inhibition of bone mineralization in FGF23-deficient mice. J. Bone Miner. Res. 29, 693-704 (2014).

58. Dempster, D. W. et al. Standardized nomenclature, symbols, and units for bone histomorphometry: a 2012 update of the report of the ASBMR Histomorphometry Nomenclature Committee. J. Bone Miner. Res. 28, 2-17 (2013).

59. Song, L. et al. Loss of wnt/beta-catenin signaling causes cell fate shift of preosteoblasts from osteoblasts to adipocytes. J. Bone Miner. Res. 27, 2344-2358 (2012). 
60. Yuan, Q. et al. Deletion of PTH rescues skeletal abnormalities and high osteopontin levels in Klotho-/- mice. PLoS Genet. 8, e1002726 (2012).

61. Wu, X. et al. Inhibition of Sca-1-positive skeletal stem cell recruitment by alendronate blunts the anabolic effects of parathyroid hormone on bone remodeling. Cell Stem Cell 7, 571-580 (2010).

62. Liu, W. et al. Effect of resorbable collagen plug on bone regeneration in rat critical-size defect model. Implant Dent. 25, 163-170 (2016).

63. Liu, W. et al. Chronic kidney disease impairs bone defect healing in rats. Sci. Rep. 6, 23041 (2016).

64. Spicer, P. P. et al. Evaluation of bone regeneration using the rat critical size calvarial defect. Nat. Protoc. 7, 1918-1929 (2012).

\section{Acknowledgements}

This work was partly supported by grants from the National Natural Science Foundation of China (NSFC 81571001, 81371173), Program for New Century Excellent Talents in University (NCET-12-0379) and Sichuan Province Youth Fund (2013JQ0017) to Q.Y.; by grants from NSFC (81321002) and 111 project of Ministry of Education of China to

Q.C. and by project of Innovative Research Team of Education Department of Sichuan Province (13TD0038) to L.Y.

\section{Author contributions}

W.L., L.Z., L.Y., Q.C. and Q.Y. designed the project; W.L., L.Z., C.Z., S.Z., J.J., L.X., N.S. and X.D. performed the experiments; W.L., L.Z., C.Z., S.Z., J.J., L.X., N.S., X.D., W.J., X.L.,
H.Z. and Q.Y. analysed the data; W.L., L.Y., Q.C. and Q.Y. wrote and edited the manuscript. All authors reviewed the manuscript.

\section{Additional Information}

Supplementary Information accompanies this paper at http://www.nature.com/ naturecommunications

Competing financial interests: The authors declare no competing financial interests.

Reprints and permission information is available online at http://npg.nature.com/ reprintsandpermissions/

How to cite this article: Liu, W. et al. GDF11 decreases bone mass by stimulating osteoclastogenesis and inhibiting osteoblast differentiation. Nat. Commun. 7:12794 doi: $10.1038 /$ ncomms12794 (2016)

\section{(c) (1)}

This work is licensed under a Creative Commons Attribution 4.0 International License. The images or other third party material in this article are included in the article's Creative Commons license, unless indicated otherwise in the credit line; if the material is not included under the Creative Commons license, users will need to obtain permission from the license holder to reproduce the material. To view a copy of this license, visit http://creativecommons.org/licenses/by/4.0/

(C) The Author(s) 2016 